\title{
VISCOSITY SOLUTIONS AND VISCOSITY SUBDERIVATIVES IN SMOOTH BANACH SPACES WITH APPLICATIONS TO METRIC REGULARITY*
}

\author{
JONATHAN M. BORWEIN ${ }^{\dagger}$ AND QIJI J. ZHU ${ }^{\ddagger}$
}

\begin{abstract}
In Gâteaux or bornologically differentiable spaces there are two natural generalizations of the concept of a Fréchet subderivative. In this paper we study the viscosity subderivative (which is the more robust of the two) and establish refined fuzzy sum rules for it in a smooth Banach space. These rules are applied to obtain comparison results for viscosity solutions of Hamilton-Jacobi equations in smooth spaces. A unified treatment of metric regularity in smooth spaces completes the paper. This illustrates the flexibility of viscosity subderivatives as a tool for analysis.
\end{abstract}

Key words. viscosity subderivative, fuzzy sum rule, viscosity solutions, Hamilton-Jacobi equations, smooth spaces, metric regularity

AMS subject classifications. 49J52, 49L25, 49J40, 49J50, 58C20

1. Introduction. It is well known that the proximal limit formula for generalized derivatives plays a crucial role in Hilbert space nonsmooth and variational analysis. The reason is twofold: many properties of a nonsmooth function are determined by the (densely existing) proximal subderivatives, and proximal subderivatives are easier to handle than various other generalized derivatives. However, the proximal derivative concept depends crucially on the analysis of nearest points and, therefore, relies heavily on the inner product structure of the underlying space. Recent research $[8,10,19]$ shows that, in fact, what is essential in this context is a "smooth" support function (rather than a nearest point) that corresponds to the "viscosity" (as opposed to the limit) subderivative concept. This makes it possible to do nonsmooth and variational analysis in smooth Banach spaces by using bornological subderivatives (compatible to the smoothness of the underlying space). Such a new technology is crucially important in studying problems on non-Fréchet-smooth spaces (see, for example, [10]).

One of the most important properties of the proximal subderivatives (and that of other generalized derivatives) is the sum rule. There is extensive research on this topic. We refer to Aubin and Frankowska [1], Clarke [11, 12], Deville and Haddad [20], Fabian [21], Ioffe [25, 26, 27, 28, 30], Jourani and Thibault [31], Kruger and Mordukhovich [32], Loewen [34], Mordukhovich [37], Mordukhovich and Shao [38], Rockafellar [41], Thibault [42], Ward and Borwein [43], and Warga [44, 45] and the references therein for sum rules for various generalized derivatives. The main purpose of this paper is to establish refined versions of the "fuzzy" sum rule given in [8] for viscosity bornological subderivatives and discuss its applications to viscosity solutions of Hamilton-Jacobi $(\mathrm{HJ})$ equations and to the metric regularity problem. Roughly speaking, the major difference between our sum rules and that of [8] is the following observation: we can have certain control on the "size" of the bornological subderivatives in the sum. This observation is new even for sum rules in finite-dimensional spaces and is important for applications (e.g., in viscosity solution theory). A crucial tool in proving our "fuzzy" sum rules is the smooth variational principle proven in [9] that requires the underlying space to have a smooth equivalent norm. In most of the following results this condition can be weakened by using the smooth variational principle proven by Deville, Godefroy, and Zizler [18]: it suffices that the space has a smooth bump function.

\footnotetext{
*Received by the editors December 16, 1994; accepted for publication (in revised form) May 6, 1995.

†Department of Mathematics and Statistics, Simon Fraser University, Burnaby, BC V5A 1S6, Canada (jborwein@cecm.sfu.ca). The research of this author was supported by the NSERC and the Shrum Endowment at Simon Fraser University.

${ }^{\ddagger}$ Department of Mathematics and Statistics, Western Michigan University, Kalamazoo, MI 49008 (zhu@mathstat.wmich.edu).
} 
Viscosity solutions were introduced by Crandall and Lions [15] to handle partial differential equations (in particular, HJ equations) that do not have any classical solution. Naturally the uniqueness of viscosity solution is one of the most important issues in the viscosity solution theory. The basic uniqueness result for finite-dimension problems was established in the pioneering paper of Crandall and Lions [15] and then developed in [13]. It is extended to Banach spaces with the Radon-Nikodým property in [16, part I]. In [19] and [20] uniqueness results are derived for HJ equations in smooth spaces with elegant short proofs using the smooth variational principle and fuzzy sum rules for viscosity subderivatives. However, the results in [19] and [20] require restrictive uniform continuity conditions to be imposed on the Hamiltonian. For HJ equations corresponding to optimal control problems involving general control equations, these uniform continuity conditions are not satisfied. By using our refined fuzzy sum rule we can prove a uniqueness theorem for an $\mathrm{HJ}$ equation under less restrictive conditions. It is applicable to infinite-horizon optimal control problems with a control equation that satisfies the usual Lipschitz condition in the state variable. We then apply this result to show that the value function of a class of infinite-horizon optimal control problems in a $\beta$-smooth space is the unique $\beta$-viscosity solution of the corresponding HJ equation. These results in particular applies to such a problem in $L^{1}$ which has a weak Hadamard smooth equivalent norm [6].

We also apply these fuzzy sum rules to give a unified treatment of metric regularity in smooth spaces. We prove a dual sufficient condition for metric regularity that is parallel to the dual conditions given in Ioffe [27] and Ginsburg and Ioffe [23] and deduce a primal condition that improves a similar condition in Borwein and Strojwas [5]. Then we show that various primal conditions discussed in $[1,2,3,5]$ can be deduced from our primary conditions. This illustrate the flexibility of viscosity subderivatives as a tool for analysis.

We introduce terminology and prove our refined fuzzy sum rules (Theorems 2.9-2.12) in $\S 2$. In $\S 3$ we discuss viscosity solutions to the $\mathrm{HJ}$ equations in smooth spaces, and in $\S 4$ we discuss metric regularity.

Finally let us remark that in bornologically differentiable spaces there are two natural generalizations of a Fréchet subderivative. It seems to us that in such spaces the viscosity subderivative (Definition 2.1) is usually the right generalization to use, rather than the limit definition.

2. Viscosity subderivatives and fuzzy sum formulae. Let $X$ be a real Banach space with closed unit ball $B$ and dual $X^{*}$. For a set $S$ in $X$, we denote its diameter by $\operatorname{diam}(S):=$ $\sup \{\|x-y\|: x, y \in S\}$. A bornology $\beta$ of $X$ is a family of closed bounded and centrally symmetric subsets of $X$ whose union is $X$, which is closed under multiplication by scalars and is directed upward (that is, the union of any two members of $\beta$ is contained in some member of $\beta$ ). We will denote by $X_{\beta}^{*}$ the dual space of $X$ endowed with the topology of uniform convergence on $\beta$-sets. The most important bornologies are those formed by all (symmetric) bounded sets (the Fréchet bornology, denoted by $F$ ), weak compact sets (the weak Hadamard bornology, denoted by $W H$ ), compact sets (the Hadamard bornology, denoted by $H$ ), and finite sets (the Gâteaux bornology, denoted by $G$ ).

We will define a convex bornology as one that also contains all convex closures of the sets in the corresponding bornology. (In particular, any finite-dimensional subspace is included in the subspace spanned by some element of the convex bornology.) Note that the convex Gâteaux bornology lies strictly between the Gâteaux and Hadamard bornology, while for the Fréchet, weak Hadamard, and Hadamard bornologies the convex and nonconvex definitions are the same.

By a function we always mean an extended-real-valued function, usually lower (upper) semicontinuous and proper (that is to say, not everywhere equal to $+\infty(-\infty)$ and nowhere 
equal to $-\infty(+\infty))$. Given a function $f$ on $X$, we say that $f$ is $\beta$-differentiable at $x$ and has a $\beta$-derivative $\nabla^{\beta} f(x)$ if $f(x)$ is finite and

$$
t^{-1}\left(f(x+t u)-f(x)-t\left\langle\nabla^{\beta} f(x), u\right\rangle\right) \rightarrow 0
$$

as $t \rightarrow 0$ uniformly in $u \in V$ for every $V \in \beta$. We say that a function $f$ is $\beta$-smooth at $x$ if $\nabla^{\beta} f: X \rightarrow X_{\beta}^{*}$ is continuous in a neighbourhood of $x$. It is not hard to check that a convex function $f$ is $\beta$-smooth at $x$ if and only if $f$ is $\beta$-differentiable on a convex neighbourhood of $x$. Now we can define $\beta$-viscosity subderivatives and superderivatives.

DEFINITION 2.1. Let $f$ be a lower semicontinuous function and $f(x)<+\infty$. We say $f$ is $\beta$-viscosity subdifferentiable and $x^{*}$ is a $\beta$-viscosity subderivative of $f$ at $x$ if there exists a locally Lipschitz function $g$ such that $g$ is $\beta$-smooth at $x, \nabla^{\beta} g(x)=x^{*}$, and $f-g$ attains a local minimum at $x$. We denote the set of all $\beta$-viscosity subderivatives of $f$ at $x$ by $D_{\beta} f(x)$.

Let $f$ be a upper semicontinuous function and $f(x)>-\infty$. We say $f$ is $\beta$-viscosity superdifferentiable and $x^{*}$ is a $\beta$-viscosity superderivative of $f$ at $x$ if there exists a locally Lipschitz function $g$ such that $g$ is $\beta$-smooth at $x, \nabla^{\beta} g(x)=x^{*}$, and $f-g$ attains a local maximum at $x$. We denote the set of all $\beta$-viscosity superderivatives of $f$ at $x$ by $D^{\beta} f(x)$.

Remark 2.2. The concepts of viscosity subderivatives and superderivatives are introduced in [19] in slightly different forms where the $\beta$-smooth function $g$ is required only to be upper semicontinuous and lower semicontinuous, respectively. We require $g$ to be locally Lipschitz because it seems more convenient for applications.

Remark 2.3. By adding a constant we may always assume that the $\beta$-smooth function $g$ in the above definition satisfies $g(x)=f(x)$.

Remark 2.4. The limit definition of the $\beta$-subdifferential $\partial_{\beta} f(x)$ of $f$ at $x$ is as follows: $x^{*} \in \partial_{\beta} f(x)$ if, for any $\varepsilon>0$ and any $V \in \beta$, there exists a $\eta>0$ such that

$$
t^{-1}(f(x+t h)-f(x))-\left\langle x^{*}, h\right\rangle>-\varepsilon \quad \forall t \in(0, \eta), h \in V .
$$

One can check that $D_{\beta} f(x) \subset \partial_{\beta} f(x)$. It is proven in [18] that $D_{F} f(x)=\partial_{F} f(x)$, provided that there exists a Lipschitz Fréchet-smooth bump function on $X$. However, the two concepts are different in general as shown by the following example.

Example 2.5. Let $f: R^{n} \rightarrow R(n \geq 2)$ be continuous, and suppose that $f$ is Gâteaux but not Fréchet (weak Hadamard) differentiable at 0 (e.g., $f: R^{2} \rightarrow R$ defined by $f(x, y)=$ $x y^{3} /\left(x^{2}+y^{4}\right)$ when $(x, y) \neq(0,0)$ and $f(0,0)=0$ at point $\left.(0,0)\right)$. Let $g(h):=-\mid f(h)-$ $f(0)-\nabla^{G} f(0) h \mid$. Then $g$ is locally uniformly continuous and

(1) $\partial_{G} g(0)=\{0\}$

(2) $D_{G} g(0)=\emptyset$.

Proof. In fact, we can directly check that $\nabla^{G} g(0)=0$; hence $\partial_{G} g(0)=\{0\}$. Since we always have $D_{G} g(0) \subset \partial_{G} g(0)$, either (2) is true or else $D_{G} g(0)=\{0\}$. In the latter case, there exists a locally Lipschitz and Gâteaux-differentiable (and therefore Fréchet-differentiable) function $k$ such that $k(0)=g(0)=0, \nabla^{G} k(0)=\nabla^{G} g(0)=0$, and $k \leq g$ in a neighbourhood of 0 . Thus,

$$
\frac{\left|f(0+h)-f(0)-\nabla^{G} f(0) h\right|}{\|h\|} \leq-\frac{k(h)-k(0)}{\|h\|} \leq \frac{|k(h)-k(0)|}{\|h\|},
$$

which implies that $f$ is Fréchet differentiable at 0 , a contradiction.

DEFINITION 2.6 (see [8]). Let $f_{1}, \ldots, f_{N}$ be lower semicontinuous functions and $E$ be a closed subset of $X$. We say that $\left(f_{1}, \ldots, f_{N}\right)$ is uniformly lower semicontinuous on $E$ if

$$
\inf _{x \in E} \sum_{n=1}^{N} f_{n}(x) \leq \lim _{\varepsilon \rightarrow 0} \inf \left\{\sum_{n=1}^{N} f_{n}\left(x_{n}\right):\left\|x_{n}-x_{m}\right\| \leq \varepsilon, x_{n}, x_{m} \in E, n, m=1, \ldots, N\right\} \text {. }
$$


We say that $\left(f_{1}, \ldots, f_{N}\right)$ is locally uniformly lower semicontinuous if, for any $x \in \cap_{n=1}^{N}$ $\operatorname{dom}\left(f_{n}\right),\left(f_{1}, \ldots, f_{N}\right)$ is uniformly lower semicontinuous on a closed ball centered at $x$.

The next useful proposition follows directly from the definition.

PROPOSITION 2.7. Let $\left(f_{1}, \ldots, f_{N}\right)$ be lower semicontinuous functions on $X$ and $E$ be a closed subset of $X$. Then the following conditions are sufficient for $\left(f_{1}, \ldots, f_{N}\right)$ to be uniformly lower semicontinuous on $E$ :

1. all but one of the functions are uniformly continuous on $E$;

2. one of the functions has compact level sets when restricted to $E$;

3. $X$ is finite dimensional and $E$ is bounded.

The following lemma, first used in [8] in a different form, is an infinite-dimensional extension of the smooth penalization result [14, Lem. 3.1].

LEMMA 2.8. Let $\left(f_{1}, \ldots, f_{N}\right)$ be lower semicontinuous functions and $E$ be a closed subset of $X$. Suppose that $\left(f_{1}, \ldots, f_{N}\right)$ is uniformly lower semicontinuous on $E$. Define, for $t>0$,

$$
M_{t}=\inf _{\left(x_{1}, \ldots, x_{N}\right) \in E^{N}}\left[\sum_{n=1}^{N} f_{n}\left(x_{n}\right)+t \sum_{n, m=1}^{N}\left\|x_{n}-x_{m}\right\|^{2}\right] .
$$

Assume that $\left(x_{1}^{t}, \ldots, x_{N}^{t}\right)$ satisfies

$$
\lim _{t \rightarrow \infty}\left(M_{t}-\left[\sum_{n=1}^{N} f_{n}\left(x_{n}^{t}\right)+t \sum_{n, m=1}^{N}\left\|x_{n}^{t}-x_{m}^{t}\right\|^{2}\right]\right)=0 .
$$

Then

(i) $\lim _{t \rightarrow \infty} t\left[\operatorname{diam}\left(\left\{x_{1}^{t}, \ldots, x_{N}^{t}\right\}\right)\right]^{2}=0$;

(ii) $\lim _{t \rightarrow \infty} M_{t}=\inf _{x \in E} \sum_{n=1}^{N} f_{n}(x)$.

Proof. Set

$$
d_{t}:=M_{t}-\left[\sum_{n=1}^{N} f_{n}\left(x_{n}^{t}\right)+t \sum_{n, m=1}^{N}\left\|x_{n}^{t}-x_{m}^{t}\right\|^{2}\right]
$$

Then $\lim _{t \rightarrow \infty} d_{t}=0$. Evidently $M_{t}$ increases with $t$ and $M_{t} \leq \inf _{x \in E} \sum_{n=1}^{N} f_{n}(x)$. Therefore, $M:=\lim _{t \rightarrow \infty} M_{t}$ exists and $M \leq \inf _{x \in E} \sum_{n=1}^{N} f_{n}(x)$. Moreover,

$$
\begin{aligned}
M_{t / 2} & \leq \sum_{n=1}^{N} f_{n}\left(x_{n}^{t}\right)+\frac{t}{2} \sum_{n, m=1}^{N}\left\|x_{n}^{t}-x_{m}^{t}\right\|^{2} \\
& =\sum_{n=1}^{N} f_{n}\left(x_{n}^{t}\right)+t \sum_{n, m=1}^{N}\left\|x_{n}^{t}-x_{m}^{t}\right\|^{2}-\frac{t}{2} \sum_{n, m=1}^{N}\left\|x_{n}^{t}-x_{m}^{t}\right\|^{2} \\
& =M_{t}-d_{t}-\frac{t}{2} \sum_{n, m=1}^{N}\left\|x_{n}^{t}-x_{m}^{t}\right\|^{2}
\end{aligned}
$$

or

$$
2\left(M_{t}-M_{t / 2}-d_{t}\right) \geq t \sum_{n, m=1}^{N}\left\|x_{n}^{t}-x_{m}^{t}\right\|^{2} \geq t\left[\operatorname{diam}\left(\left\{x_{1}^{t}, \ldots, x_{N}^{t}\right\}\right)\right]^{2}
$$

Thus,

$$
\lim _{t \rightarrow \infty} t\left[\operatorname{diam}\left(\left\{x_{1}^{t}, \ldots, x_{N}^{t}\right\}\right)\right]^{2}=0
$$


It remains to show that $M \geq \inf _{x \in E} \sum_{n=1}^{N} f_{n}(x)$. To do so, observe that $\left\|x_{n}^{t}-x_{m}^{t}\right\| \rightarrow 0$ when $t \rightarrow \infty$ and $\left(f_{1}, \ldots, f_{N}\right)$ is uniformly lower semicontinuous on $E$. Therefore,

$$
\begin{aligned}
M & =\lim _{t \rightarrow \infty}\left(\sum_{n=1}^{N} f_{n}\left(x_{n}^{t}\right)+t \sum_{n, m=1}^{N}\left\|x_{n}^{t}-x_{m}^{t}\right\|^{2}\right) \\
& \geq \liminf _{t \rightarrow \infty} \sum_{n=1}^{N} f_{n}\left(x_{n}^{t}\right) \geq \inf _{x \in E} \sum_{n=1}^{N} f_{n}(x) .
\end{aligned}
$$

The next theorem is a refined fuzzy sum rule for the sum of several functions at its minimum. It refines [8, Prop. 4] in that it gives a bound for the $\beta$-subderivatives in the fuzzy sum. This bound is new even for fuzzy sum rules in finite-dimensional spaces and is important in application to the uniqueness result for $\mathrm{HJ}$ equations.

THEOREM 2.9. Let $X$ be a Banach space with an equivalent $\beta$-smooth norm and $f_{1}, \ldots, f_{N}$ be lower semicontinuous functions on $X$. Suppose that $\left(f_{1}, \ldots, f_{N}\right)$ is locally uniformly lower semicontinuous and $\sum_{n=1}^{N} f_{n}$ attains a local minimum at $x$. Then, for any $\varepsilon>0$, there exist $x_{n} \in x+\varepsilon B$ and $x_{n}^{*} \in D_{\beta} f_{n}\left(x_{n}\right), n=1, \ldots, N$, such that $\left|f_{n}\left(x_{n}\right)-f_{n}(x)\right|<\varepsilon$, $\left\|x_{n}^{*}\right\| \operatorname{diam}\left(\left\{x_{1}, \ldots, x_{N}\right\}\right)<\varepsilon, n=1,2, \ldots, N$, and

$$
\left\|\sum_{n=1}^{N} x_{n}^{*}\right\|<\varepsilon .
$$

Proof. Let $\varepsilon>0$ be given. Taking a smaller $\varepsilon$ if necessary, we may assume that $\left(f_{1}, \ldots, f_{N}\right)$ is uniformly lower semicontinuous on $x+\varepsilon B$ and that $\sum_{n=1}^{N} f_{n}$ attains a minimum at $x$ over $x+\varepsilon B$. Define

$$
w_{t}\left(y_{1}, \ldots, y_{N}\right):=\sum_{n=1}^{N} f_{n}\left(y_{n}\right)+t \sum_{n, m=1}^{N}\left\|y_{n}-y_{m}\right\|^{2} .
$$

Then, by Lemma 2.8 ,

$$
\begin{aligned}
& \lim _{t \rightarrow \infty} \inf \left\{w_{t}\left(y_{1}, \ldots, y_{N}\right): y_{n} \in x+\varepsilon B, n=1, \ldots, N\right\} \\
= & \inf _{y \in x+\varepsilon B} \sum_{n=1}^{N} f_{n}(y)=\sum_{n=1}^{N} f_{n}(x)=w_{t}(x, \ldots, x) .
\end{aligned}
$$

Choose $t_{i} \rightarrow \infty$ such that

$$
w_{t_{i}}(x, \ldots, x)<\inf \left\{w_{t_{i}}\left(y_{1}, \ldots, y_{N}\right): y_{n} \in x+\varepsilon B, n=1, \ldots, N\right\}+\frac{\varepsilon^{2}}{N i} .
$$

Then by the Borwein-Preiss smooth variational principle [9], for each $i$ there exist a $\beta$-smooth function $\phi_{i}$ and $x_{n}^{i}, n=1, \ldots, N$, such that $w_{t_{i}}+\phi_{i}$ attains a local minimum at $\left(x_{1}^{i}, \ldots, x_{N}^{i}\right)$, $\left\|\nabla^{\beta} \phi_{i}\right\|<\varepsilon / N,\left\|x_{n}^{i}-x\right\|<\varepsilon / i$, and

$$
w_{t_{i}}\left(x_{1}^{i}, \ldots, x_{N}^{i}\right)<\inf \left\{w_{t_{i}}\left(y_{1}, \ldots, y_{N}\right): y_{n} \in x+\varepsilon B, n=1, \ldots, N\right\}+\frac{\varepsilon^{2}}{N i} .
$$

For each $n$, the function

$$
y \rightarrow w_{t_{i}}\left(x_{1}^{i}, \ldots, x_{n-1}^{i}, y, x_{n+1}^{i}, \ldots, x_{N}^{i}\right)+\phi_{i}\left(x_{1}^{i}, \ldots, x_{n-1}^{i}, y, x_{n+1}^{i}, \ldots, x_{N}^{i}\right)
$$


attains a local minimum at $y=x_{n}^{i}$. Thus

$$
x_{n_{i}}^{*}:=-\nabla_{x_{n}}^{\beta} \phi_{i}\left(x_{1}^{i}, \ldots, x_{N}^{i}\right)-2 t_{i} \sum_{m=1}^{N} \nabla^{\beta}\|\cdot\|^{2}\left(x_{n}^{i}-x_{m}^{i}\right) \in D_{\beta} f_{n}\left(x_{n}^{i}\right) .
$$

Summing $x_{n_{i}}^{*}, n=1, \ldots, N$, yields

$$
\sum_{n=1}^{N} x_{n_{i}}^{*}=-\sum_{n=1}^{N} \nabla_{x_{n}}^{\beta} \phi_{i}\left(x_{1}^{i}, \ldots, x_{N}^{i}\right)-2 t_{i} \sum_{n=1}^{N} \sum_{m=1}^{N} \nabla^{\beta}\|\cdot\|^{2}\left(x_{n}^{i}-x_{m}^{i}\right) .
$$

Observing that $\left\|-\sum_{n=1}^{N} \nabla_{x_{n}}^{\beta} \phi_{i}\left(x_{1}^{i}, \ldots, x_{N}^{i}\right)\right\| \leq \varepsilon$ and

$$
\nabla^{\beta}\|\cdot\|^{2}\left(x_{n}^{i}-x_{m}^{i}\right)+\nabla^{\beta}\|\cdot\|^{2}\left(x_{m}^{i}-x_{n}^{i}\right)=0
$$

so that the double sum in the previous inclusion vanishes, we obtain

$$
\left\|\sum_{n=1}^{N} x_{n_{i}}^{*}\right\| \leq \varepsilon
$$

By (1) and (2)

$$
\sum_{n=1}^{N} f_{n}(x)=\lim _{i \rightarrow \infty} \sum_{n=1}^{N} f_{n}\left(x_{n}^{i}\right) .
$$

Since $f_{n}, n=1, \ldots, N$, are lower semicontinuous

$$
\lim _{i \rightarrow \infty} f_{n}\left(x_{n}^{i}\right)=f_{n}(x), \quad n=1, \ldots, N .
$$

Moreover, Lemma 2.8 and (2) imply

$$
\lim _{i \rightarrow \infty} t_{i}\left[\operatorname{diam}\left(\left\{x_{1}^{i}, \ldots, x_{N}^{i}\right\}\right)\right]^{2}=0 .
$$

Since $\nabla^{\beta}\|\cdot\|^{2}(x)$ is bounded by $2\|x\|$, combining (4) and (3) yields

$$
\lim _{i \rightarrow \infty}\left\|x_{n_{i}}^{*}\right\| \operatorname{diam}\left(\left\{x_{1}^{i}, \ldots, x_{N}^{i}\right\}\right)=0 \text { for } n=1, \ldots, N .
$$

Thus, when $i$ is sufficiently large,

$$
\left\|x_{n}^{i}-x\right\|<\varepsilon, \quad\left|f_{n}\left(x_{n}^{i}\right)-f_{n}(x)\right|<\varepsilon, \quad \text { and }\left\|x_{n_{i}}^{*}\right\| \operatorname{diam}\left(\left\{x_{1}^{i}, \ldots, x_{N}^{i}\right\}\right)<\varepsilon
$$

for $n=1,2, \ldots, N$. It remains to take $x_{n}=x_{n}^{i}$ and $x_{n}^{*}=x_{n_{i}}^{*}, n=1, \ldots, N$.

The next three theorems provide general forms of sum rules for $\beta$-viscosity subderivatives.

THEOREM 2.10. Let $X$ be a Banach space with an equivalent $\beta$-smooth norm. Let $f_{1}, \ldots, f_{N}$ be lower semicontinuous functions on $X$, with $x \in \cap_{n=1}^{N} \operatorname{dom}\left(f_{n}\right)$. Then, for any $x^{*} \in D_{\beta}\left(\sum_{n=1}^{N} f_{n}\right)(x), \varepsilon>0$, and any weak-star neighbourhood $V$ of 0 in $X^{*}$, there exist $x_{n} \in x+\varepsilon B, x_{n}^{*} \in D_{\beta} f_{n}\left(x_{n}\right), n=1, \ldots, N$, such that $\left|f_{n}\left(x_{n}\right)-f_{n}(x)\right|<\varepsilon$, $\left\|x_{n}^{*}\right\| \operatorname{diam}\left(\left\{x_{1}, \ldots, x_{N}\right\}\right)<\varepsilon, n=1,2, \ldots, N$, and

$$
x^{*} \in \sum_{n=1}^{N} x_{n}^{*}+V .
$$


Proof. Let $\varepsilon>0$ and $V$ be a weak-star neighbourhood of 0 in $X^{*}$. Fix $r>0, L$ a finite-dimensional subspace of $X$ containing $x$, and $V_{\beta}$ a $\beta$-neighbourhood of 0 in $X_{\beta}^{*}$ such that $V_{\beta}+L^{\perp}+r B_{X^{*}} \subset V$. Since $x^{*} \in D_{\beta}\left(\sum_{n=1}^{N} f_{n}\right)(x)$, there exists a locally Lipschitz function $g$ such that $g$ is $\beta$ smooth at $x$ with $\nabla^{\beta} g(x)=x^{*}$ and $\sum_{n=1}^{N} f_{n}-g$ attains a local minimum at $x$. Choose $0<\eta<\min (\varepsilon, r)$ such that $\|y-x\|<\eta<\varepsilon$ implies that $\nabla^{\beta} g(x)-\nabla^{\beta} g(y) \in V_{\beta}$, and let $\delta_{L}$ be the indicator function of $L$. Then $\sum_{n=1}^{N} f_{n}-g+\delta_{L}$ attains a local minimum at $x$. By Proposition $2.7\left(f_{1}, \ldots, f_{N},-g, \delta_{L}\right)$ is locally uniformly lower semicontinuous. Applying Theorem 2.9 yields the existence of $x_{n}, n=1, \ldots, N+2$, such that $\left\|x_{n}-x\right\|<\eta<\varepsilon, n=1, \ldots, N+2, x_{n}^{*} \in D_{\beta} f\left(x_{n}\right), n=1, \ldots, N, x_{N+1}^{*}=-\nabla^{\beta} g\left(x_{N+1}\right)$, and $x_{N+2}^{*} \in D_{\beta} \delta_{L}\left(x_{N+2}\right)$ satisfying the conclusion of Theorem 2.9, i.e., $\left|f_{n}\left(x_{n}\right)-f_{n}(x)\right|<$ $\eta<\varepsilon,\left\|x_{n}^{*}\right\| \operatorname{diam}\left(\left\{x_{1}, \ldots, x_{N}\right\}\right) \leq\left\|x_{n}^{*}\right\| \operatorname{diam}\left(\left\{x_{1}, \ldots, x_{N+2}\right\}\right)<\eta<\varepsilon$ for $n=1, \ldots, N$, $\left|\delta_{L}\left(x_{N+2}\right)-\delta_{L}(x)\right|<\eta$, i.e., $x_{N+2} \in L$, and

$$
\sum_{n=1}^{N} x_{n}^{*}-\nabla^{\beta} g\left(x_{N+1}\right)+x_{N+2}^{*} \in r B_{X^{*}} .
$$

Note that $D_{\beta} \delta_{L}\left(x_{N+2}\right)=L^{\perp}$ and $x^{*}-\nabla^{\beta} g\left(x_{N+1}\right) \in V_{\beta}$.

Using the method in [8], we can prove the following stronger result.

THEOREM 2.11. Let $\beta$ be a convex bornology and $X$ be a Banach space with an equivalent $\beta$-smooth norm. Let $f_{1}, \ldots, f_{N}$ be lower semicontinuous functions and $x \in \cap_{n=1}^{N} \operatorname{dom}\left(f_{n}\right)$. Then, for any $x^{*} \in \partial_{\beta}\left(\sum_{n=1}^{N} f_{n}\right)(x), \varepsilon>0$, and any weak-star neighbourhood $V$ of 0 in $X^{*}$, there exist $x_{n} \in x+\varepsilon B, x_{n}^{*} \in D_{\beta} f_{n}\left(x_{n}\right), n=1, \ldots, N$, such that $\left|f_{n}\left(x_{n}\right)-f_{n}(x)\right|<\varepsilon$, $\left\|x_{n}^{*}\right\| \operatorname{diam}\left(\left\{x_{1}, \ldots, x_{N}\right\}\right)<\varepsilon, n=1,2, \ldots, N$, and

$$
x^{*} \in \sum_{n=1}^{N} x_{n}^{*}+V .
$$

Proof. Let $\varepsilon>0$ and $V$ be a weak-star neighbourhood of 0 in $X^{*}$. Fix $r>0$ and $L$ a finite-dimensional subspace of $X$ containing $x$ such that $L^{\perp}+2 r B_{X^{*}} \subset V$. Let $x^{*} \in$ $\partial_{\beta}\left(\sum_{n=1}^{N} f_{n}\right)(x)$. Then, for any $K \in \beta$,

$$
\liminf _{t \rightarrow 0^{+}} \inf _{h \in t K} t^{-1}\left[\sum_{n=1}^{N}\left(f_{n}(x+h)-f_{n}(x)\right)-\left\langle x^{*}, h\right\rangle\right] \geq 0 .
$$

Choose a $K \in \beta$ containing the intersection of $L$ with a ball around zero. Then (5) in particular implies that

$$
\liminf _{t \rightarrow 0^{+}} \inf _{h \in t B \cap L} t^{-1}\left[\sum_{n=1}^{N}\left(f_{n}(x+h)-f_{n}(x)\right)-\left\langle x^{*}, h\right\rangle\right] \geq 0 .
$$

Since $L$ is a finite-dimensional space, this is equivalent to

$$
\liminf _{\|y-x\| \rightarrow 0} \inf _{y-x \in L}\|y-x\|^{-1}\left[\sum_{n=1}^{N}\left(f_{n}(y)-f_{n}(x)\right)-\left\langle x^{*}, y-x\right\rangle\right] \geq 0 .
$$

Thus, there exists $\eta<r$ such that the function

$$
y \rightarrow \sum_{n=1}^{N} f_{n}(y)-\left\langle x^{*}, y\right\rangle+r\|y-x\|+\delta_{L}(y)
$$

attains a minimum over $y \in x+\eta B$ at $y=x$. 
By Proposition 2.7, the mapping $y \rightarrow\left(f_{1}(y), \ldots, f_{N}(y),-\left\langle x^{*}, y\right\rangle, r\|y-x\|, \delta_{L}(y)\right)$ is locally uniformly lower semicontinuous. Applying Theorem 2.9 yields that there exist $x_{n}, n=1, \ldots, N+3$, with $\left\|x_{n}-x\right\|<\eta<\varepsilon, n=1, \ldots, N+3 ; x_{n}^{*} \in D_{\beta} f\left(x_{n}\right), n=$ $1, \ldots, N ; x_{N+1}^{*}=-x^{*}, x_{N+2}^{*} \in r D_{\beta}\left\|x_{N+2}-x\right\|$, and $x_{N+3}^{*} \in D_{\beta} \delta_{L}\left(x_{N+3}\right)$ such that $\left|f_{n}\left(x_{n}\right)-f_{n}(x)\right|<\eta<\varepsilon,\left\|x_{n}^{*}\right\| \operatorname{diam}\left(\left\{x_{1}, \ldots, x_{N}\right\}\right) \leq\left\|x_{n}^{*}\right\| \operatorname{diam}\left(\left\{x_{1}, \ldots, x_{N+3}\right\}\right)<\eta<\varepsilon$ for $n=1, \ldots, N,\left|\delta_{L}\left(x_{N+3}\right)-\delta_{L}(x)\right|<\eta$, i.e., $x_{N+3} \in L$ and

$$
\sum_{n=1}^{N} x_{n}^{*}-x^{*}+x_{N+2}^{*}+x_{N+3}^{*} \in r B_{X^{*}}
$$

Observing that $D_{\beta} \delta_{L}\left(x_{N+3}\right)=L^{\perp}$ and $r D_{\beta}\left\|x_{N+2}-x\right\| \subset r B_{X^{*}}$, we obtain

$$
x^{*} \in \sum_{n=1}^{N} x_{n}^{*}+L^{\perp}+2 r B_{X^{*}} \subset \sum_{n=1}^{N} x_{n}^{*}+V .
$$

THEOREM 2.12. Let $X$ be a Banach space with an equivalent $\beta$-smooth norm. Let $f_{1}, \ldots, f_{N}$ be lower semicontinuous functions, with all but one of $f_{n}, n=1, \ldots, N$, locally uniformly continuous and $x \in \cap_{n=1}^{N} \operatorname{dom}\left(f_{n}\right)$. Then, for any $x^{*} \in D_{\beta}\left(\sum_{n=1}^{N} f_{n}\right)(x), \varepsilon>0$, and any $\beta$-neighbourhood $V$ of 0 in $X_{\beta}^{*}$, there exist $x_{n} \in x+\varepsilon B, x_{n}^{*} \in D_{\beta} f_{n}\left(x_{n}\right), n=1, \ldots, N$, such that $\left|f_{n}\left(x_{n}\right)-f_{n}(x)\right|<\varepsilon,\left\|x_{n}^{*}\right\| \operatorname{diam}\left(\left\{x_{1}, \ldots, x_{N}\right\}\right)<\varepsilon, n=1,2, \ldots, N$, and

$$
x^{*} \in \sum_{n=1}^{N} x_{n}^{*}+V .
$$

Proof. Let $\varepsilon>0$ and $V$ be a neighbourhood of 0 in $X_{\beta}^{*}$. Let $r>0, U$ be a neighbourhood of $0 \in X_{\beta}^{*}$ such that $U+r B_{X^{*}} \subset V$. Let $x^{*} \in D_{\beta}\left(\sum_{n=1}^{N} f_{n}\right)(x)$. Then there exists a $\beta$ smooth function $g$ such that $\nabla^{\beta} g(x)=x^{*}$ and $\sum_{n=1}^{N} f_{n}-g$ attains a local minimum at $x$. Choose $0<\eta<\varepsilon$ such that $\|y-x\|<\eta<\varepsilon$ implies that $\nabla^{\beta} g(x)-\nabla^{\beta} g(y) \in U$. By Proposition $2.7\left(f_{1}, \ldots, f_{N},-g\right)$ is locally uniformly lower semicontinuous. Applying Theorem 2.9 yields that there exist $x_{n}, n=1, \ldots, N+1$, with $\left\|x_{n}-x\right\|<\eta<\varepsilon, n=1, \ldots, N+1$, $x_{n}^{*} \in D_{\beta} f_{n}\left(x_{n}\right), n=1, \ldots, N$, and $x_{N+1}^{*}=-\nabla^{\beta} g\left(x_{N+1}\right)$ such that $\left|f_{n}\left(x_{n}\right)-f_{n}(x)\right|<\eta<$ $\varepsilon,\left\|x_{n}^{*}\right\| \operatorname{diam}\left(\left\{x_{1}, \ldots, x_{N}\right\}\right)<\varepsilon, n=1, \ldots, N$, and

$$
\left\|\sum_{n=1}^{N} x_{n}^{*}-\nabla^{\beta} g\left(x_{N+1}\right)\right\|<r .
$$

Thus,

$$
\begin{aligned}
x^{*} & \in \sum_{n=1}^{N} x_{n}^{*}+\nabla^{\beta} g(x)-\nabla^{\beta} g\left(x_{N+1}\right)+r B_{X^{*}} \\
& \subset \sum_{n=1}^{N} x_{n}^{*}+U+r B_{X^{*}} \subset \sum_{n=1}^{N} x_{n}^{*}+V .
\end{aligned}
$$

Remark 2.13. As in [9] we call a lower semicontinuous function $f: X \rightarrow[-\infty, \infty]$ $s$-Hölder subdifferentiable at $x(s \in(0,1])$ with subderivative $x^{*} \in X^{*}$ if $f$ is finite at $x$ and there exists a positive constant $C_{x}$ such that

$$
f(y)-f(x)-\left\langle x^{*}, y-x\right\rangle \geq-C_{x}\|y-x\|^{1+s}
$$


for all $y$ in a neighbourhood of $x$. We denote the set of $s$-Hölder subderivatives of $f$ at $x$ by $\partial^{H(s)} f(x)$. When $s=1$, such subderivatives are called Lipschitz smooth, and in Hilbert space they coincide with Rockafellar's proximal subderivatives, written $\partial^{\pi} f(x)$ in [40]. Then all the above statements and proofs will still hold true in a Banach space with a power modulus of smoothness $t^{p}$ [33] if we replace $\beta$ with $H(p-1)$.

Remark 2.14. By the same argument as in [18, $\S$ VIII, Lem. 1.3] we can prove the following result.

LEMMA 2.15. Let $X$ be a Banach space that admits a bump function which is Lipschitzian and $\beta$-smooth. Then there exist a function $d: X \rightarrow R^{+}$and a scalar $K>1$ such that

i) $d$ is bounded, Lipschitzian on $X$, and $\beta$-smooth on $X \backslash\{0\}$.

ii) $\|x\| \leq d(x) \leq K\|x\|$ if $\|x\| \leq 1$ and $d(x)=2$ if $\|x\| \geq 1$.

Then, observing that we can replace the $\|\cdot\|^{2}$ term in Lemma 2.8 and Theorem 2.9 and their proofs with $d(\cdot)^{2}$ and using the Deville-Godefroy-Zizler smooth variational principle (cf. $[18,39]$ ) in place of the Borwein-Preiss smooth variational principle, the condition that $X$ has a $\beta$-smooth norm can be replaced by the weaker condition that $X$ has a $\beta$-smooth Lipschitzian bump function.

Remark 2.16. Ioffe $[25,26]$ named Banach spaces that have the fuzzy sum rules for lower semicontinuous functions for the $\varepsilon$-Fréchet-subderivative and the $\varepsilon$-Dini-subderivative trustworthy $(T)$ and weak trustworthy $(W T)$ spaces, respectively. He proved that if a Banach space has a Fréchet- or Gâteaux-smooth norm, then it has property $(T)$ or $(W T)$. It is also known that $(T)$ or $(W T)$ is equivalent to the dense $\varepsilon$-Fréchet-subdifferentiability $(S)$ or dense $\varepsilon$-Dini-subdifferentiability (WS) of lower semicontinuous functions [21]. In this spirit we will call a Banach space $\beta$-trustworthy $\left(T_{\beta}\right)$ if it has the property stated in the conclusion of Theorem 2.10 (without requiring the bound on the $\beta$-subderivatives in the sum) and say that a Banach space is a (strong) dense $\beta$-subdifferentiability space if, for any lower semicontinuous function $f$, the $\operatorname{set}((x, f(x)) \in \operatorname{graph}(f)) x \in \operatorname{dom}(f)$ for which $D_{\beta} f(x) \neq \emptyset$ is dense in the (graph) domain of $f$. We will use notations $S_{\beta}^{+}$and $S_{\beta}$ for strong $\beta$-subdifferentiability and $\beta$ subdifferentiability spaces, respectively. We will denote the properties "has a $\beta$-smooth norm" and "has a $\beta$-smooth Lipschitz bump function" by $H_{\beta}$ and $H_{\beta}^{-}$, respectively. Then Theorem 2.10 and Remark 2.14 tell us that $H_{\beta} \Longrightarrow H_{\beta}^{-} \Longrightarrow T_{\beta}$. It is obvious that $S_{\beta}^{+} \Longrightarrow S_{\beta}$. Let $f$ be a lower semicontinuous function in a $T_{\beta}$ space. Then, for any $x \in \operatorname{dom}(f)$, applying the $T_{\beta}$ property to $f+\delta_{\{x\}}$ yields $S_{\beta}^{+}$. Therefore, we have

$$
H_{\beta} \Longrightarrow H_{\beta}^{-} \Longrightarrow T_{\beta} \Longrightarrow S_{\beta}^{+} \Longrightarrow S_{\beta}
$$

In [21] it is shown that $T \Longleftrightarrow S$ and $W T \Longleftrightarrow W S$. Using similar arguments we can show that $T_{\beta} \Longleftrightarrow S_{\beta}^{+}$. However, it is not clear if $S_{\beta} \Longleftrightarrow S_{\beta}^{+}$. Since conditions $H_{\beta}$ and $H_{\beta}^{-}$are easier to check than $S_{\beta}$ and $S_{\beta}^{+}$and can yield a bound on the $\beta$-subderivatives in the fuzzy sum, we will not pursue this topic further here.

Adapting the arguments in $[7,8]$ and using Theorem 2.9 we can obtain corresponding viscosity versions of the sequential limit formulae for the $g$-subdifferential of a function and the $g$-normal cone of a closed set. In the following theorems, $\partial_{g}, \partial_{c}, N_{g}$, and $N_{c}$ signify the $g$-subdifferential, Clarke generalized gradient, $g$-normal cone, and Clarke normal cone, respectively, and we refer the reader to [8] and the references therein for their definitions.

THEOREM 2.17. Let $X$ be a Banach space with an equivalent $\beta$-smooth norm. Let $f$ be a lower semicontinuous proper function on $X$. Then for any $x \in X$

$$
\partial_{g} f(x)=\mathrm{cl}^{*} \bigcup_{k=1}^{\infty}\left\{\mathrm{w}^{*}-\lim _{n \rightarrow \infty} x_{n}^{*}: x_{n}^{*} \in D_{\beta}^{k} f\left(x_{n}\right), x_{n} \rightarrow_{f} x\right\}
$$


and

$$
\partial_{c} f(x)=\mathrm{cl}^{*} c o \bigcup_{k=1}^{\infty}\left\{\mathrm{w}^{*}-\lim _{n \rightarrow \infty} x_{n}^{*}: x_{n}^{*} \in D_{\beta}^{k} f\left(x_{n}\right), x_{n} \rightarrow_{f} x\right\}+\partial_{c}^{\infty} f(x) .
$$

where $D_{\beta}^{k} f(x)$ is the subset of $D_{\beta} f(x)$ for which the support function in the definition has a Lipschitz constant no greater than $k$.

THEOREM 2.18. Let $X$ be a Banach space with an equivalent $\beta$-smooth norm. Let $S$ be a closed subset of $X$. Then for any $x \in S$

$$
N_{g}(S, x)=\mathrm{cl}^{*} \bigcup_{k=1}^{\infty}\left\{\mathrm{w}^{*}-\lim _{n \rightarrow \infty} x_{n}^{*}: x_{n}^{*} \in k D_{\beta} d\left(S, x_{n}\right), x_{n} \rightarrow_{s} x\right\}
$$

and

$$
N_{c}(S, x)=\mathrm{cl}^{*} c o \bigcup_{k=1}^{\infty}\left\{\mathrm{w}^{*}-\lim _{n \rightarrow \infty} x_{n}^{*}: x_{n}^{*} \in k D_{\beta} d\left(S, x_{n}\right), x_{n} \rightarrow_{s} x\right\}
$$

In the following discussion we will use the formulae given in the above theorems as equivalent definitions for the $g$-normal cone in a $\beta$-smooth space. These formulae also have corresponding $s$-Hölder versions. For details see [10].

3. Viscosity solutions to $\mathbf{H J}$ equations. Consider the partial differential equation

$$
F(x, u, D u)=0 .
$$

This equation encompasses the usual $\mathrm{HJ}$ equation associated with the optimal value function of certain optimal control problems. In general, (6) does not have a classical solution. Viscosity solutions were introduced by Crandall and Lions [15] to replace classical solutions. The original definition of viscosity solutions (cf. $[15,16]$ ) is based on the Fréchet-subderivative. In [19], $\beta$-viscosity solutions are defined for problems on non-Fréchet-smooth spaces. We recall this definition below.

DEFINITION 3.1 (see [19]). Let $X$ be a Banach space with an equivalent $\beta$-smooth norm. A function $u: X \rightarrow R$ is a $\beta$-viscosity subsolution of (6) if $u$ is upper semicontinuous and, for every $x \in X$ and every $x^{*} \in D^{\beta} u(x)$,

$$
F\left(x, u(x), x^{*}\right) \leq 0 .
$$

A function $u: X \rightarrow R$ is a $\beta$-viscosity supersolution of (6) if $u$ is lower semicontinuous and, for every $x \in X$ and every $x^{*} \in D_{\beta} u(x)$,

$$
F\left(x, u(x), x^{*}\right) \geq 0 .
$$

A continuous function $u$ is called $a \beta$-viscosity solution if $u$ is both a $\beta$-viscosity subsolution and $a \beta$-viscosity supersolution.

Now we prove the main result of this section.

THEOREM 3.2. Let $X$ be a Banach space with an equivalent $\beta$-smooth norm. Suppose that $\gamma>0, F\left(x, u, x^{*}\right)=\gamma u+H\left(x, x^{*}\right)$, and $H: X \times X_{\beta}^{*} \rightarrow R$ satisfy the following assumption:

(A) for any $x_{1}, x_{2} \in X$ and $x_{1}^{*}, x_{2}^{*} \in X_{\beta}^{*}$,

$$
\left|H\left(x_{1}, x_{1}^{*}\right)-H\left(x_{2}, x_{2}^{*}\right)\right| \leq \omega\left(x_{1}-x_{2}, x_{1}^{*}-x_{2}^{*}\right)+M \max \left(\left\|x_{1}^{*}\right\|,\left\|x_{2}^{*}\right\|\right)\left\|x_{1}-x_{2}\right\|,
$$

where $M>0$ is a constant and $\omega: X \times X_{\beta}^{*} \rightarrow R$ is a continuous function with $\omega(0,0)=0$. 
Let $u$ and $v$ be two uniformly continuous functions such that $v$ is bounded below and $u$ is bounded above. If $u$ is a $\beta$-viscosity subsolution of (6) and $v$ is a $\beta$-viscosity supersolution of (6), then $u \leq v$.

Proof. Let $\varepsilon>0$ be an arbitrary positive number. By assumption (A) there exist $\eta \in(0, \varepsilon)$ and a neighbourhood $V$ of 0 in $X_{\beta}^{*}$ such that $\left\|x_{1}-x_{2}\right\|<2 \eta$ and $x_{1}^{*}-x_{2}^{*} \in V$ implies that

$$
\left|H\left(x_{1}, x_{1}^{*}\right)-H\left(x_{2}, x_{2}^{*}\right)\right|<\varepsilon+M \max \left(\left\|x_{1}^{*}\right\|,\left\|x_{2}^{*}\right\|\right)\left\|x_{1}-x_{2}\right\| .
$$

The function $v-u$ is uniformly continuous and bounded below. By the smooth variational principle there exist $x \in X$ and $x^{*} \in D_{\beta}(v-u)(x)$ such that $x^{*}+\frac{1}{2} V \subset V$ and $(v-u)(x)<$ $\inf _{X}(v-u)+\varepsilon$. By Theorem 2.12 with $f_{1}=v$ and $f_{2}=-u$, there exist $x_{1}, x_{2} \in X$, $x_{1}^{*} \in D_{\beta} v\left(x_{1}\right)$, and $x_{2}^{*} \in D^{\beta} u\left(x_{2}\right)$ satisfying

(i) $\left\|x_{1}-x\right\|<\eta$ and $\left\|x_{2}-x\right\|<\eta$;

(ii) $\left|v\left(x_{1}\right)-v(x)\right|<\varepsilon$ and $\left|u\left(x_{2}\right)-u(x)\right|<\varepsilon$;

(iii) $\left\|x_{1}^{*}\left|\left\||| x_{1}-x_{2}\right\|<\varepsilon\right.\right.$ and $\left\|x_{2}^{*}\left|\left\||| x_{1}-x_{2}\right\|<\varepsilon\right.\right.$;

(iv) $x_{1}^{*}-x_{2}^{*}-x^{*} \in \frac{1}{2} V$.

Since the function $u$ is a viscosity subsolution of (6) we have

$$
F\left(x_{1}, u\left(x_{1}\right), x_{1}^{*}\right)=\gamma u\left(x_{1}\right)+H\left(x_{1}, x_{1}^{*}\right) \leq 0 .
$$

Similarly

$$
F\left(x_{2}, v\left(x_{2}\right), x_{2}^{*}\right)=\gamma v\left(x_{2}\right)+H\left(x_{2}, x_{2}^{*}\right) \geq 0 .
$$

Therefore, observing that $\left\|x_{1}-x_{2}\right\|<2 \eta$ and $x_{1}^{*}-x_{2}^{*} \in V$,

$$
\begin{aligned}
\inf _{X}(v-u) & >(v-u)(x)-\varepsilon>v\left(x_{2}\right)-u\left(x_{1}\right)-3 \varepsilon \\
& \geq \gamma^{-1}\left[H\left(x_{1}, x_{1}^{*}\right)-H\left(x_{2}, x_{2}^{*}\right)\right]-3 \varepsilon \\
& \geq-\gamma^{-1}\left[\varepsilon+M \max \left(\left\|x_{1}^{*}\right\|,\left\|x_{2}^{*}\right\|\right)\left\|x_{1}-x_{2}\right\|\right]-3 \varepsilon \\
& \geq-\left[\gamma^{-1}(1+M)+3\right] \varepsilon .
\end{aligned}
$$

As $\varepsilon$ is arbitrary, $\inf _{X}(v-u) \geq 0$.

COROLLARY 3.3. Under the assumptions of Theorem 3.2 any uniformly continuous bounded $\beta$-viscosity solution to (6) is unique.

Remark 3.4. Condition (A) is significantly weaker than the uniform continuity conditions imposed in $[19,20]$. This allows us to apply Corollary 3.3 to the HJ equation corresponding to a general optimal control problem $P(x)$ in the sequel. In [16, part I], a uniqueness result is established for $\mathrm{HJ}$ equations in Banach spaces with the Radon-Nikodým property under somewhat weaker (but rather technical) conditions. How much we can weaken our requirements for the Hamiltonian in a $\beta$-smooth space is an interesting problem that we will not pursue further in this paper.

Remark 3.5. Definition 3.1 is slightly different from the original definition in [19] in that we require $X$ to be $\beta$-smooth. We make this modification to avoid the following pathological examples.

Example 3.6. Let $X$ be a $\beta^{\prime}$-differentiable Banach space with a nowhere $\beta$-differentiable norm $\|\cdot\|$. Consider the case of (6) where $F$ is the constant function 1 . Then obviously this equation does not have any $\beta^{\prime}$-viscosity solution, but $u(x)=(\|x\|+2) /(\|x\|+1)$ is a uniformly continuous bounded $\beta$-viscosity solution in the sense of [19, Def. III.2]. To see this, observe that $u$ is trivially a supersolution. Since $u(x)$ is convex and nowhere $\beta$-differentiable 
(otherwise $\|x\|=(2-u(x)) /(u(x)-1)$ would be $\beta$-differentiable), $D^{\beta} u(x)=\emptyset$ for all $x \in X$. Thus, $u$ is also a $\beta$-viscosity subsolution and, therefore, a $\beta$-viscosity solution in the sense of [19, Def. III.2].

Example 3.7. In Banach space $X$ consider

$$
V(x)+\|D V(x)\|=0 .
$$

Assume that the norm $\|\cdot\|$ of $X$ is nowhere $\beta$-differentiable and $X$ has an equivalent $\beta^{\prime}$-smooth norm. Then, in the sense of [19, Def. III.2],

1. $V=0$ is the unique uniformly continuous bounded $\beta^{\prime}$-viscosity solution of (7);

2. $V(x)=(\|x\|+2) /(\|x\|+1)$ is a uniformly continuous bounded $\beta$-viscosity solution of (7).

Proof. Step 1. If $V$ is a uniformly continuous bounded $\beta^{\prime}$-viscosity solution of (7), then $u$ is nonnegative. In fact, if $-\Delta=\inf _{X} V<0$, then since $X$ has an equivalent $\beta^{\prime}$-smooth norm, by the Borwein-Preiss smooth variational principle, there exists an $x \in X$ and a $p \in X^{*}$ such that

$$
V(x)<\inf _{X} V+\frac{\Delta}{2}
$$

and

$$
p \in D_{\beta^{\prime}} V(x), \quad\|p\|<\frac{\Delta}{2} .
$$

This implies

$$
V(x)+\|p\|<0
$$

so that $V$ is not a $\beta^{\prime}$-viscosity supersolution of (7), which is absurd.

Step 2. If $V$ is a nonnegative $\beta^{\prime}$-viscosity solution of (7), then $V$ is a nonnegative $\beta^{\prime}$ viscosity subsolution of (7). Thus, $D^{\beta^{\prime}} V(x)=\{0\}$ whenever it is nonempty. By [10, Thm. 6.3] $u$ is a constant. It is then obvious that $V$ must be the zero function. This completes the proof of 1 .

Step 3. We prove 2. It is trivial to observe that $V(x)=(\|x\|+2) /(\|x\|+1)$ is a $\beta$-viscosity supersolution of (7). Since $V(x)$ is convex and nowhere $\beta$-differentiable, $D^{\beta} V(x)=\emptyset$ for all $x \in X$. Thus, $V$ is also a $\beta$-viscosity subsolution and, therefore, a $\beta$-viscosity solution of (7) according to [19, Def. III.2].

Note that (7) is the HJ equation corresponding to the optimal control problem

$$
\begin{aligned}
& \operatorname{minimize} J(x, u):=\int_{0}^{\infty} e^{-s} f(x(s), u(s)) d s \\
& \text { subject to } \dot{x}(s)=u(s), \\
& \qquad x(0)=x, u(s) \in B_{X},
\end{aligned}
$$

with $f=0$. Since the optimal value function for this problem is identically 0 , it is reasonable to expect 0 to be the unique viscosity solution of (7). Thus, it is reasonable to require $X$ to be $\beta$-smooth in the definition of $\beta$-viscosity solutions.

As concrete examples of the spaces in Examples 3.6 and 3.7,

a. the sup norm of $C[0,1]$ is Gâteaux smooth but nowhere weak Hadamard differentiable [6]. 
b. $L_{1}[0,1]$ or $l_{1}(N)$ has an equivalent weak Hadamard smooth norm [6] but is not Asplund and, therefore, has a nowhere Fréchet-differentiable norm. Moreover, $L_{1}[0,1]$ fails to have the Radon-Nikodým property.

Remark 3.8. Let $X$ be a Banach space with a $\beta$-smooth equivalent norm. Then this norm is also $\beta^{\prime}$-smooth for any $\beta^{\prime} \subset \beta$. Thus, in Theorem 3.2 and Corollary 3.3 we can always reduce the requirement on the smoothness of the space by using a smaller bornology. However, the price to pay is that we have to impose stronger continuity conditions on $F$.

Let $X$ be a Banach space with a $\beta$-smooth norm and $U$ be a metric space. We now consider the following optimal control problem in $X$ :

$$
\begin{gathered}
P(x): \quad \text { minimize } J(x, u):=\int_{0}^{\infty} e^{-\gamma s} f(x(s), u(s)) d s \\
\text { subject to } \dot{x}(s)=g(x(s), u(s)), \\
x(0)=x, u \in \mathcal{U},
\end{gathered}
$$

where $g: X \times U \rightarrow R$ is continuous and Lipschitz in $x$ uniformly in $U$ and there exists $K \in \beta$ such that $g(x, U) \subset K$ for all $x \in X, f: X \times U \rightarrow R$ is continuous, bounded, continuous in $x$ uniformly in $U$, and

$$
\mathcal{U}:=\{u: u \text { is measurable and } u(t) \in U \text { for } t \in[0, \infty) \text { a. e. }\} .
$$

Under our assumptions, for given $x \in X$ and $u \in \mathcal{U}, \dot{x}(s)=g(x(s), u(s)), x(0)=x$ has a unique solution defined on $[0, \infty)$, denoted by $x(s, x, u)$.

Since $f$ is bounded, the problem is well defined. We denote the value function of $P(x)$ by $V(x)$. Then we have the following.

THEOREM 3.9 (dynamic programming principle). For any $t>0$,

$$
V(x)=\inf _{u \in \mathcal{U}}\left\{\int_{0}^{t} e^{-\gamma s} f(x(s, x, u), u(s)) d s+e^{-\gamma t} V(x(t, x, u))\right\} .
$$

This theorem is standard; for a proof see, for example, [22].

Define $H: X \times X^{*} \rightarrow R$ by

$$
H(x, p)=\sup _{u \in U}\{-\langle p, g(x, u)\rangle-f(x, u)\} .
$$

We prove the following theorem.

THEOREM 3.10. $V$ is the unique $\beta$-viscosity solution of the HJ equation

$$
\gamma V(x)+H(x, D V(x))=0 .
$$

Proof. Since $f$ is bounded, so is $V$. Using Gronwall's inequality, since $f$ is continuous in $x$ uniformly in $U$, we can show that $V$ is uniformly continuous. One can directly check that $H(x, p)$ satisfies assumption (A). Therefore, uniqueness is a direct consequence of Corollary 3.3. We need only to show that $V$ is a $\beta$-viscosity solution of (8).

a. Subsolution. Let $y$ be an element of $X$ such that $p \in D^{\beta} V(y)$. Then there exists a $\beta$-smooth locally Lipschitz function $w$ such that $\nabla^{\beta} w(y)=p, y$ is a (local) maximum of $V-w$, and $0=(V-w)(y)$. Note that $w$ depends only on $y$ and $p$ and is fixed in the subsequent discussion. By the dynamic programming principle, for any $u \in \mathcal{U}$ and $t>0$,

$$
w(y)=V(y) \leq \int_{0}^{t} e^{-\gamma s} f(x(s, y, u), u(s)) d s+e^{-\gamma t} V(x(t, y, u)) .
$$


Since $w$ is $\beta$-smooth and locally Lipschitz at $x$, there exists $\eta>0$ such that $w$ is Lipschitz and $\nabla^{\beta} w$ exist in $y+\eta B$. Therefore, $w$ is at least Hadamard differentiable and $\nabla^{\beta} w=\nabla^{H} w$ in $y+\eta B$. Observing that $\{x(s, y, u): s \in[0,1]\}$ is compact, when $t>0$ is sufficiently small, we have

$$
\frac{d}{d t}\left[e^{-\gamma t} w(x(t, y, u))\right]=-e^{-\gamma t} \gamma w(x(t, y, u))+e^{-\gamma t}\left\langle\nabla^{\beta} w(x(t, y, u)), g(x(t, y, u), u(t))\right\rangle \text {. }
$$

Thus, we can write (9) as

$$
\begin{gathered}
t^{-1} \int_{0}^{t} e^{-\gamma s}\left[\gamma w(x(s, y, u))-\left\langle\nabla^{\beta} w(x(s, y, u)), g(x(s, y, u), u(s))\right\rangle\right. \\
-f(x(s, y, u), u(s))] d s \leq 0 .
\end{gathered}
$$

Fixing an arbitrary $v \in U$ and setting $u(s)=v$ for all $s \in[0, t]$ yield

$t^{-1} \int_{0}^{t} e^{-\gamma s}\left[\gamma w(x(s, y, u))-\left\langle\nabla^{\beta} w(x(s, y, u)), g(x(s, y, u), v)\right\rangle-f(x(s, y, u), v)\right] d s \leq 0$.

Taking limits when $t \rightarrow 0$, observing that the integrand is continuous in $s$ and $x(0, y, u)=y$, we obtain

$$
\gamma V(y)-\langle p, g(y, v)\rangle-f(y, v)=\gamma w(y)-\left\langle\nabla^{\beta} w(y), g(y, v)\right\rangle-f(y, v) \leq 0 .
$$

Therefore,

$$
\gamma V(y)+H(y, p) \leq 0
$$

that is to say, $V$ is a $\beta$-viscosity subsolution of (8).

b. Supersolution. Now let $y$ be an element of $X$ such that $p \in D_{\beta} V(y)$. Then there exists a $\beta$-smooth function $w$ such that $\nabla^{\beta} w(y)=p, y$ is a (local) minimum of $V-w$, and $0=(V-w)(y)$. By the dynamic programming principle, for each integer $i$, there exists $u^{i} \in \mathcal{U}$ such that

$$
\begin{aligned}
w(y)+\frac{1}{i^{2}} & =V(y)+\frac{1}{i^{2}} \\
& \geq \int_{0}^{1 / i} e^{-\gamma s} f\left(x\left(s, y, u^{i}\right), u^{i}(s)\right) d s+e^{-\gamma / i} V\left(x\left(\frac{1}{i}, y, u^{i}\right)\right) .
\end{aligned}
$$

Arguments similar to those in the previous paragraph yield

$$
\begin{aligned}
& \frac{1}{i}+i \int_{0}^{1 / i} e^{-\gamma s} {\left[\gamma w\left(x\left(s, y, u^{i}\right)\right)\right.} \\
&\left.-\left\langle\nabla^{\beta} w\left(x\left(s, y, u^{i}\right)\right), g\left(x\left(s, y, u^{i}\right), u^{i}(s)\right)\right\rangle-f\left(x\left(s, y, u^{i}\right), u^{i}(s)\right)\right] d s \geq 0 .
\end{aligned}
$$

We rewrite this inequality as

$$
\gamma w(y)+i \int_{0}^{1 / i}\left[-\left\langle\nabla^{\beta} w(y), g\left(y, u^{i}(s)\right)\right\rangle-f\left(y, u^{i}(s)\right)\right] d s \geq h(i),
$$

where

$$
h(i)=-\frac{1}{i}+h_{1}(i)+h_{2}(i)+h_{3}(i)
$$

and the $h_{j}$ 's are defined as follows:

$$
h_{1}(i):=\gamma w(y)-i \int_{0}^{1 / i} e^{-\gamma s} \gamma w\left(x\left(s, y, u^{i}\right)\right) d s
$$




$$
h_{2}(i):=i \int_{0}^{1 / i}\left[e^{-\gamma s}\left\langle\nabla^{\beta} w\left(x\left(s, y, u^{i}\right)\right), g\left(x\left(s, y, u^{i}\right), u^{i}(s)\right)\right\rangle-\left\langle\nabla^{\beta} w(y), g\left(y, u^{i}(s)\right)\right\rangle\right] d s,
$$

and

$$
h_{3}(i):=i \int_{0}^{1 / i}\left[e^{-\gamma s} f\left(x\left(s, y, u^{i}\right), u^{i}(s)\right)-f\left(y, u^{i}(s)\right)\right] d s .
$$

It is obvious that

$$
-\left\langle\nabla^{\beta} w(y), g\left(y, u^{i}(s)\right)\right\rangle-f\left(y, u^{i}(s)\right) \leq H(y, p)
$$

and, therefore,

$$
\gamma V(y)+H(y, p) \geq h(i)
$$

Since

$$
\sup \left\{\left\|x\left(s, y, u^{i}\right)-y\right\|: s \in\left[0, \frac{1}{i}\right]\right\} \rightarrow 0
$$

when $i \rightarrow \infty, \lim _{i \rightarrow \infty} h_{1}(i)=\lim _{i \rightarrow \infty} h_{3}(i)=0$. Observing that $g$ is Lipschitz in $x$ uniformly in $U$ and $g\left(y, u^{i}(s)\right)$ is in the $\beta$-set $K$, the $\beta$-smoothness of $w$ at $y$ yields $\lim _{i \rightarrow \infty} h_{2}(i)=0$. Therefore, $\lim _{i \rightarrow \infty} h(i)=0$. Sending $i$ to infinity in (11), we obtain that $V$ is a $\beta$-viscosity supersolution of (8) and, hence, a $\beta$-viscosity solution of (8).

4. Metric regularity. Metric regularity is closely related to the open mapping property and plays an important role in studying exact penalization and necessary conditions for constrained minimization problems. In this section we apply the fuzzy sum rules derived in $\S 2$ to derive a necessary condition for metric regularity to fail; contraposition produces a sufficient condition for a function to be metrically regular at a given point. We deduce a dual condition parallel to the dual conditions given in Ioffe [27] and Ginsburg and Ioffe [23] and a $\beta$-smooth space version of the primal condition discussed in Borwein and Strojwas [5]. Many authors have discussed primal sufficient conditions. The condition that we give below (Theorem 4.9) appears to be the weakest (in $\beta$-smooth spaces) up to now. Various primal conditions discussed in $[1,2,3,5]$ are deduced as corollaries. To avoid complications in the notations we consider metric regularity for a single-valued function with respect to a closed set. This is in fact an entirely general formulation (see [1] and [29]). There are many discussions about regularity of multifunctions (see, for example, $[31,35]$ and the references therein). We will indicate at the end of this section how to handle multifunctions with our results. We recall the following definition of metric regularity.

DEFINITION 4.1. Let $X$ and $Y$ be Banach spaces. One says that $h: X \rightarrow Y$ is regular with respect to $S$ at $x_{0}$ if there exist positive constants $\varepsilon$ and $K$ such that, for all $x \in S \cap\left(x_{0}+\varepsilon B\right)$ and $\xi \in h\left(x_{0}\right)+\varepsilon B$,

$$
d\left(x, S \cap h^{-1}(\xi)\right) \leq K\|h(x)-\xi\| .
$$

DEFINITION 4.2. Let $X$ and $Y$ be Banach spaces with $\beta$-smooth norms, $S$ be a closed subset of $X$, and $h: X \rightarrow Y$ be differentiable at $x_{0}$. We say that $x_{0}$ is an extremal with respect to $(h, S)$ if, for any $\eta>0$, there exist a unit vector $y^{*}, y \in\left(x_{0}+\eta B\right) \cap S$, and a constant $L$ such that

$$
0 \in\left(\nabla h\left(x_{0}\right)\right)^{*} y^{*}+L D_{\beta} d(y, S)+\eta B_{X^{*}}
$$


The next theorem shows that a function is not metrically regular at a point corresponding to the fuzzy extremal condition described above at the given point. The essential part of the proof is an application of the Ekeland variational principle that was first introduced in [24] for handling metric regularity problems and now becomes quite standard. Before stating the theorem, let us recall that a mapping $f: X \rightarrow Y$ is called strictly differentiable at $x$, provided that $f$ is (Fréchet) differentiable at $x$, for each $v$,

$$
\lim _{x^{\prime} \rightarrow x, t \rightarrow 0^{+}}\left\|\frac{f\left(x^{\prime}+t v\right)-f\left(x^{\prime}\right)}{t}-\langle\nabla f(x), v\rangle\right\|=0,
$$

and the convergence is uniform for $v \in B_{X}$. Note that $f$ is strictly differentiable at $x$ implies that $f$ is locally Lipschitz at $x$.

THEOREM 4.3. Let $X$ and $Y$ be Banach spaces with $\beta$-smooth norms, $S$ be a closed subset of $X$, and $x_{0} \in S$. Assume that $h: X \rightarrow Y$ is strictly differentiable at $x_{0}$. Then $h$ is not regular with respect to $S$ at $x_{0}$ implies that $x_{0}$ is an extremal with respect to $(h, S)$.

Proof. Let $\eta$ be an arbitrary positive constant in $(0,1)$. Since $h$ is strictly differentiable at $x_{0}$, for any $d \in B_{X}$, there exists $\eta_{1}<\eta / 4$ such that $\left\|x-x_{0}\right\|<\eta_{1}$ and $t \in\left(0, \eta_{1}\right)$ implies that

$$
\frac{h(x+t d)-h(x)}{t}-\nabla h\left(x_{0}\right) d \in \frac{\eta}{2} B_{Y}
$$

and

$$
\left\|h(x)-h\left(x_{0}\right)\right\|<\frac{\eta^{2}}{128}
$$

Suppose $h$ is not regular with respect to $S$ at $x_{0}$. Then there exist an $s \in S \cap\left(x_{0}+\frac{\eta_{1}}{4} B\right)$ and a $\xi \in h\left(x_{0}\right)+\frac{\eta^{2}}{128} B$ such that

$$
d\left(s, S \cap h^{-1}(\xi)\right)>\frac{1}{\eta}\|h(s)-\xi\| .
$$

By inequality (13) $d\left(s, S \cap h^{-1}(\xi)\right)>0$ and, therefore, $\|h(s)-\xi\|>0$. Apply Ekeland's principle with

$$
\begin{array}{cl}
f(x):=\|h(x)-\xi\|, & \varepsilon:=\|h(s)-\xi\| \leq \frac{\eta^{2}}{64} \\
\lambda:=\frac{\eta}{8}, & \bar{x}:=s .
\end{array}
$$

Then there exist $v \in S$ such that

1. $\|s-v\| \leq \frac{\eta}{8}$.

2. $v$ minimizes

$$
x \rightarrow\|h(x)-\xi\|+\frac{\varepsilon}{\lambda}\|x-v\|
$$

on $S$.

Since $h$ is strictly differentiable at $x_{0}$, it is locally Lipschitz at $x_{0}$. Let $L^{\prime}$ be a local Lipschitz constant for $h$ at $x_{0}$. Then $L:=L^{\prime}+1$ is a local Lipschitz constant for $x \rightarrow\|h(x)\|+\frac{\varepsilon}{\lambda}\|x-v\|$. Thus, using the penalization result of Clarke [11] we have 
$2^{\prime} . \quad v$ locally minimizes

$$
x \rightarrow\|h(x)-\xi\|+\frac{\varepsilon}{\lambda}\|x-v\|+L \cdot d(x, S) .
$$

By Theorem 2.9 there exist $y, z$, and $w$ such that

$$
\begin{aligned}
0 & \in D_{\beta}\|h(\cdot)-\xi\|(y)+\frac{\varepsilon}{\lambda} D_{\beta}\|w-v\|+L D_{\beta} d(z, S)+\frac{\eta}{8} B_{X^{*}} \\
& \subset D_{\beta}\|h(\cdot)-\xi\|(y)+L D_{\beta} d(z, S)+\frac{\eta}{4} B_{X^{*}},
\end{aligned}
$$

where $\|y-v\|<\frac{\eta_{1}}{4}$ and $\|z-v\|<\frac{\eta_{1}}{4}$ and, therefore, $\left\|y-x_{0}\right\|<\eta_{1}$ and $\left\|z-x_{0}\right\|<\eta_{1}$. Without loss of generality we may assume that $y$ were chosen close enough to $s$ such that $\|h(y)-\xi\|>0$. Then, for any $d \in B$, when $t$ is small enough, $y_{t}^{*}(d):=\nabla^{\beta}\|\|.(h(y+t d)-\xi)$ exists. Since $\left\|y_{t}^{*}(d)\right\|=1$, by (12), for any $d \in B_{X}$, when $t$ is sufficiently small,

$$
\begin{aligned}
\frac{\eta}{2} & \geq\left\langle y_{t}^{*}(d), \frac{h(y+t d)-h(y)}{t}-\nabla h\left(x_{0}\right) d\right\rangle \\
& =\left\langle y_{t}^{*}(d), \frac{(h(y+t d)-\xi)-(h(y)-\xi)}{t}\right\rangle-\left\langle y_{t}^{*}(d), \nabla h\left(x_{0}\right) d\right\rangle \\
& \geq \frac{\|h(y+t d)-\xi\|-\|h(y)-\xi\|}{t}-\left\langle y_{t}^{*}(d), \nabla h\left(x_{0}\right) d\right\rangle .
\end{aligned}
$$

By inclusion (14) there exists $z^{*} \in L D_{\beta} d(z, S)$ such that $z^{*}=-u^{*}+v^{*}$, where $u^{*} \in$ $D_{\beta}\|h()-.\xi\|(y)$ and $v^{*} \in \frac{\eta}{4} B_{X^{*}}$. Observe that, for a fixed $d$, there exists a $t_{d}$ such that when $t \in\left(0, t_{d}\right)$,

$$
\frac{\|h(y+t d)-\xi\|-\|h(y)-\xi\|}{t}>\left\langle u^{*}, d\right\rangle-\frac{\eta}{4} \geq\left\langle z^{*}, d\right\rangle-\frac{\eta}{2} .
$$

Combining this with (15) we obtain that when $t \in\left(0, t_{d}\right)$

$$
\eta \geq-\left\langle y_{t}^{*}(d), \nabla h\left(x_{0}\right) d\right\rangle-\left\langle z^{*}, d\right\rangle .
$$

Denote $y^{*}=\nabla^{\beta}\|\|.(h(y)-\xi)$. Then $y_{t}^{*}(d)=\nabla^{\beta}\|\|.(h(y+t d)-\xi) \rightarrow w^{*} y^{*}$ as $t \rightarrow 0^{+}$ and $\left\|y^{*}\right\|=1$. (In fact, $y_{t}^{*}(d)$ converges to $y^{*}$ in $Y_{\beta}^{*}$.) Taking limits in the above inequality as $t \rightarrow 0^{+}$yields

$$
\eta \geq-\left\langle y^{*}, \nabla h\left(x_{0}\right) d\right\rangle-\left\langle z^{*}, d\right\rangle .
$$

Since this is true for all $d \in B$, we obtain

$$
\left(\nabla h\left(x_{0}\right)\right)^{*} y^{*}+z^{*} \in \eta B_{X^{*}}
$$

or

$$
0 \in\left(\nabla h\left(x_{0}\right)\right)^{*} y^{*}+L D_{\beta} d(y, S)+\eta B_{X^{*}},
$$

as was to be shown.

We now turn to some sufficient conditions for $h$ to be regular with respect to $S$ at $x_{0}$. We consider a dual condition first. To do so we need the following definitions. 
DEFINITION 4.4. Let $X$ be a Banach space with a $\beta$-smooth norm and $S$ be a closed subset of $X$. We define the $\beta$-normal cone (denoted by $N_{\beta}(x, S)$ ) and the $\beta$-tangent cone (denoted by $T_{\beta}(x, S)$ ) of $S$ at $x \in S$ by

$$
N_{\beta}(x, S):=\bigcup_{L>0} L \cdot D_{\beta} d(x, S)
$$

and

$$
T_{\beta}(x, S):=\left(N_{\beta}(x, S)\right)^{0} .
$$

Here we adopt the convention that $(\emptyset)^{0}=X$.

Remark 4.5. Recall that the contingent cone $T_{b}(x, S)$ and the Clarke tangent cone $T_{c}(x, S)$ of $S$ at $x \in S$ are defined by

$$
T_{b}(x, S):=\left\{u: \liminf _{t \rightarrow 0^{+}} \frac{d(x+t u, S)}{t}=0\right\}
$$

and

$$
T_{c}(x, S):=\left\{u: \limsup _{t \rightarrow 0^{+}, y \rightarrow x} \frac{d(y+t u, S)}{t}=0\right\},
$$

respectively. It is easy to see that $T_{c}(x, S) \subset T_{b}(x, S) \subset T_{\beta}(x, S)$ for any bornology $\beta$. Note also that $N_{\beta}(x, S)$ need not be closed. Also, it is known [8] that $T_{c}(x, S)=\left(N_{g}(x, S)\right)^{0}$.

The next definition is a $\beta$-normal version of the finite-codimension condition defined in [23]. In a $\beta$-smooth space this condition is less restrictive than the definition in [23].

DEFINITION 4.6. Let $X$ be a Banach space with an equivalent $\beta$-smooth norm and $Y$ be a Banach space. We say that $f: X \rightarrow Y$ has the $\beta$-finite codimension property with respect to $S$ at $x_{0}$ if there is a weak-star closed subspace $V^{*} \subset Y^{*}$ of finite codimension and constants $\varepsilon, c>0$ such that if

(1) $x \in S$ and $\left\|x-x_{0}\right\|<\varepsilon$,

(2) $x^{*} \in N_{\beta}(x, S)$,

(3) $\left\|y^{*}\right\|=1, d\left(y^{*}, V^{*}\right)<\varepsilon$, then

$$
\left\|\left(\nabla f\left(x_{0}\right)\right)^{*} y^{*}+x^{*}\right\| \geq c .
$$

The next theorem can be deduced from [23, Thm. 2.7]. We give a self-contained proof by using Theorem 4.3.

THEOREM 4.7. Let $X$ and $Y$ be Banach spaces with $\beta$-smooth norms and $S$ be a closed subset of $X$. Assume that $h$ is strictly differentiable at $x_{0}$ and has the $\beta$-finite-codimension property with respect to $S$ at $x_{0}$. Then

$$
c l \nabla h\left(x_{0}\right) T_{c}\left(x_{0}, S\right)=Y
$$

implies that $h$ is regular with respect to $S$.

Proof. In light of Theorem 4.3 we need only to show that $x_{0}$ is not an extremal with respect to $(h, S)$. Assume on the contrary that $x_{0}$ is an extremal with respect to $(h, S)$. Then, for each $n$, there exist a unit vector $y_{n}^{*}$ and $y_{n} \in\left(x_{0}+\frac{1}{n} B\right) \cap S$ such that

$$
0 \in\left(\nabla h\left(x_{0}\right)\right)^{*} y_{n}^{*}+L D_{\beta} d\left(y_{n}, S\right)+\frac{1}{n} B_{X^{*}}
$$


We may assume that $y_{n}^{*} \rightarrow_{w^{*}} y^{*}$. Then by the definition of the $g$-normal cone

$$
-\left(\nabla h\left(x_{0}\right)\right)^{*} y^{*} \in N_{g}\left(x_{0}, S\right) .
$$

In other words, $y^{*}$ is normal to $\nabla h\left(x_{0}\right) T_{c}\left(x_{0}, S\right)$ (because $\left.T_{c}\left(x_{0}, S\right)=N_{g}\left(x_{0}, S\right)^{0}\right)$, which, together with (16), yields $y^{*}=0$. Since $h$ has the $\beta$-finite-codimension property with respect to $S$ at $x_{0}$, there exists a weak-star closed subspace $V^{*}$ of $Y^{*}$ such that $h, S$, and $V^{*}$ satisfy the conditions in Definition 4.4. Write $Y^{*}=V^{*}+W^{*}$, where $W^{*}$ is a finite-dimensional space with $V^{*} \cap W^{*}=\{0\}$ and $y_{n}^{*}=v_{n}^{*}+w_{n}^{*}$ with $v_{n}^{*} \in V^{*}$ and $w_{n}^{*} \in W^{*}$. Since $W^{*}$ is finite dimensional and $\left\|w_{n}^{*}\right\| \leq\left\|y_{n}^{*}\right\|=1$, without loss of generality we may assume that $w_{n}^{*} \rightarrow w^{*}$. Since $y^{*}=0$, we have $w^{*}=0$. Thus, $d\left(V^{*}, y_{n}^{*}\right) \rightarrow 0$. Observe that (17) implies that, for each $n$, there exists an $e_{n}^{*} \in \frac{1}{n} B_{X^{*}}$ such that

$$
u_{n}^{*}:=-\left(\nabla h\left(x_{0}\right)\right)^{*} y_{n}^{*}+e_{n}^{*} \in L D_{\beta} d\left(y_{n}, S\right) \subset N_{\beta}\left(y_{n}, S\right) .
$$

When $n$ is sufficiently large, the $\beta$-finite-codimension property of $h$ with respect to $S$ at $x_{0}$ implies that

$$
0<c \leq\left\|\left(\nabla h\left(x_{0}\right)\right)^{*} y_{n}^{*}+u_{n}^{*}\right\|=\left\|e_{n}^{*}\right\| \leq \frac{1}{n} \quad \forall n,
$$

which is a contradiction.

Remark 4.8. Since $N_{\beta}(x, S) \subset N_{g}(x, S)$, a function $f$ which satisfies the codimension condition in [23] also satisfies the $\beta$-finite-codimension property. Thus, the $\beta$-finitecodimension property is less restrictive than the codimension condition in [23]. However, we should note that the result in [23] was proven without the smoothness assumption on the underlying space.

Now we turn to a $\beta$-smooth space version of the primal condition discussed in [5].

THEOREM 4.9. Let $X$ and $Y$ be Banach spaces with $\beta$-smooth norms and $S$ be a closed subset of $X$. Assume that $h: X \rightarrow Y$ is strictly differentiable at $x_{0}$. Suppose that

(i) $\operatorname{cl} \nabla h\left(x_{0}\right) T_{c}\left(x_{0}, S\right)=Y$,

(ii) there exists a nonempty compact set $K \subset Y$ and positive numbers $\alpha, \gamma$, and $\eta$ such that $\alpha<\gamma$ and, for all $\left\|x-x_{0}\right\| \leq \eta$,

$$
\gamma B_{Y} \subset \operatorname{cl}\left\{\nabla h\left(x_{0}\right)\left(T_{\beta}(x, S) \cap B_{X}\right)+\alpha B_{Y}\right\}+K .
$$

Then $h$ is regular with respect to $S$ at $x_{0}$.

Proof. We need only to show that the condition of this theorem implies that of Theorem 4.7. Suppose that conditions (i) and (ii) are satisfied. By [4, Lem. 2.1] we may assume that $K$ is finite dimensional. Let $V:=\operatorname{span}(K)$ and

$$
V^{*}:=\left\{y^{*} \in Y^{*}: y^{*} \perp V\right\} .
$$

Then $V^{*}$ is of finite codimension. Let

$$
\varepsilon:=\min \left\{\eta, \frac{\gamma-\alpha}{2 \sup \{\|k\|: k \in K\}}\right\}
$$

and $c:=\gamma-\varepsilon \sup \{\|k\|: k \in K\}-\alpha$. Consider $x, x^{*}$, and $y^{*}$ satisfying (1), (2), and (3) in Definition 4.12. Let $\xi$ be an arbitrary element of $\gamma B_{Y}$. Then by condition (ii), for any $\alpha^{\prime}>\alpha$, there exist $u \in T_{\beta}(x, S) \cap B_{X}, b \in B_{Y}$, and $k \in K$ such that

$$
\xi=\nabla h\left(x_{0}\right) u+\alpha^{\prime} b+k .
$$


Then

$$
\begin{aligned}
\left\langle y^{*},-\xi\right\rangle & =\left\langle\left(\nabla h\left(x_{0}\right)\right)^{*} y^{*},-u\right\rangle+\alpha^{\prime}\left\langle y^{*},-b\right\rangle+\left\langle y^{*},-k\right\rangle \\
& =\left\langle\left(\nabla h\left(x_{0}\right)\right)^{*} y^{*}+x^{*},-u\right\rangle+\left\langle x^{*}, u\right\rangle+\alpha^{\prime}\left\langle y^{*},-b\right\rangle+\left\langle y^{*},-k\right\rangle \\
& \leq\left\|\left(\nabla h\left(x_{0}\right)\right)^{*} y^{*}+x^{*}\right\|+\alpha^{\prime}+\varepsilon \sup \{\|k\|: k \in K\} .
\end{aligned}
$$

Taking the supremum on the left-hand side and letting $\alpha^{\prime} \rightarrow \alpha$ lead to

$$
\gamma \leq\left\|\left(\nabla h\left(x_{0}\right)\right)^{*} y^{*}+x^{*}\right\|+\alpha+\varepsilon \sup \{\|k\|: k \in K\}
$$

or

$$
c \leq\left\|\left(\nabla h\left(x_{0}\right)\right)^{*} y^{*}+x^{*}\right\| .
$$

Remark 4.10. Since $T_{c}(x, S) \subset T_{b}(x, S) \subset T_{\beta}(x, S)$, the condition of this theorem is weaker than that of [5, Thm. 4.1] in $\beta$-smooth spaces. We should note that the results of [5, Thm. 4.1] are proven without the $\beta$-smoothness assumption. The same remark also applies to the corollaries below.

As shown in Borwein and Strojwas [5], in a $\beta$-smooth space, conditions (i) and (ii) in Theorem 4.9 are weaker than the sufficient conditions for regularity given in Borwein [3] for the cases where (i) $Y$ is a finite-dimensional space, (ii) $S$ is convex, and (iii) $S$ is epi-Lipschitz-like (see [5] for the definition). Therefore we have the following corollary.

COROLLARY 4.11 (see $[3,5]$ ). Let $X$ and $Y$ be Banach spaces with $\beta$-smooth norms and $S$ be a closed subset of $X$. Assume that $h: X \rightarrow Y$ is strictly differentiable at $x_{0} \in S$. Then $h$ is regular with respect to $S$ at $x_{0}$, provided that one of the following conditions is satisfied:

a. $S$ is convex and $0 \in \operatorname{core} \nabla h\left(x_{0}\right)\left(S-x_{0}\right)$.

b. $S$ is epi-Lipschitz-like and $\nabla h\left(x_{0}\right) T_{c}\left(x_{0}, S\right)=Y$.

c. $Y$ is finite dimensional and $\nabla h\left(x_{0}\right) T_{c}\left(x_{0}, S\right)=Y$.

Next we show that the sufficient condition for regularity in terms of uniform sleekness of the set $S$ given by Aubin and Frankowska [1] is also a direct consequence of Theorem 4.9. First we recall the definitions of sleekness and uniform sleekness.

DEFINITION 4.12. We say $S$ is $\beta$-sleek at $x$ if

$$
\liminf _{y \rightarrow x} T_{\beta}(y, S) \supset T_{b}(x, S) .
$$

We say $S$ is uniformly $\beta$-sleek at $x$ if, for $u \in T_{b}(x, S) \cap B$,

$$
\lim _{y \rightarrow x} d\left(u, T_{\beta}(y, S)\right)=0
$$

uniformly.

Remark 4.13. (a) By Remark $4.5 \beta$-sleekness is weaker than sleekness defined in [1].

(b) Borwein and Ioffe [8] have shown that

$$
T_{c}(x, S) \supset \liminf _{y \rightarrow x} T_{\beta}(y, S) .
$$

It is well known that $T_{c}(x, S) \subset T_{b}(x, S)$. Thus, if $S$ is $\beta$-sleek at $x$, then $T_{c}(x, S)=T_{b}(x, S)$ (i.e., $S$ is $b$-tangentially regular at $x$ ).

COROLLARY 4.14. Let $X$ and $Y$ be Banach spaces with $\beta$-smooth norms and $S$ be a closed subset of $X$. Assume that $S$ is uniformly $\beta$-sleek at $x_{0}$ and $h: X \rightarrow Y$ is strictly differentiable at $x_{0} \in S$. Then

$$
\nabla h\left(x_{0}\right) T_{c}\left(x_{0}, S\right)=Y
$$

implies that $h$ is regular with respect to $S$ at $x_{0}$. 
Proof. We need only to show that $h$ and $S$ satisfy condition (ii) in Theorem 4.9. By the Robinson-Ursescu theorem (see [1]) there exists a $\gamma>0$ such that

$$
\gamma B_{Y} \subset \nabla h\left(x_{0}\right)\left(T_{c}\left(x_{0}, S\right) \cap \frac{1}{2} B_{X}\right) .
$$

Take an $\varepsilon<\frac{1}{2}$ such that $\alpha:=\varepsilon\left\|\nabla h\left(x_{0}\right)\right\|<\gamma$. The uniform sleekness of $S$ at $x_{0}$ implies that there exists an $\eta$ such that $\left\|x-x_{0}\right\| \leq \eta$ implies that

$$
T_{c}\left(x_{0}, S\right) \cap \frac{1}{2} B_{X} \subset T_{\beta}(x, S) \cap B_{X}+\varepsilon B_{X} .
$$

Therefore

$$
\gamma B_{Y} \subset \nabla h\left(x_{0}\right)\left(T_{c}\left(x_{0}, S\right) \cap \frac{1}{2} B_{X}\right) \subset \operatorname{cl}\left\{\nabla h\left(x_{0}\right)\left(T_{\beta}(x, S) \cap B_{X}\right)+\alpha B_{Y}\right\},
$$

as was to be shown.

Recently Azé and Chou [2] derived a primal sufficient condition in terms of equi-circatangent cones. It turns out this condition can also be deduced directly from Theorem 4.9.

DEFINITION 4.15 (see [2]). A cone $K \subset X$ is said to be equi-circatangent to $S$ at $x \in S$ if

$$
\lim _{t \rightarrow 0^{+}, y \rightarrow s^{x}} e\left(K \cap B_{X}, t^{-1}(S-y)\right)=0,
$$

where $e(A, S):=\inf \left\{\lambda>0: A \subset S+\lambda B_{X}\right\}$.

The primal condition discussed in [2] (as specialized for single-valued functions) can be stated as the following corollary.

COROLLARY 4.16. Let $X$ and $Y$ be Banach spaces with $\beta$-smooth norms and $S$ be a closed convex subset of $X$. Assume that $h: X \rightarrow Y$ is strictly differentiable at $x_{0}$. Let $K$ be an equi-circatangent cone of $S$ at $x_{0}$. Suppose that

$$
\nabla h\left(x_{0}\right) K=Y \text {. }
$$

Then $h$ is regular with respect to $S$ at $x_{0}$.

Proof. We show that the conditions of this corollary imply those of Theorem 4.9. Condition (i) is obvious. To show condition (ii), invoke the Robinson-Ursescu theorem: there exists a positive constant $\gamma$ such that

$$
\gamma B_{X} \subset \nabla h\left(x_{0}\right)\left(K \cap \frac{1}{2} B_{X}\right) .
$$

By the definition of $K$ there exists $\eta>0$ such that, for all $t \in(0, \eta)$ and $x \in\left(x_{0}+\eta B_{X}\right) \cap S$,

$$
K \cap B_{X} \subset \frac{S-x}{t}+\varepsilon B_{X}
$$

where $\varepsilon:=\min \left(\frac{\gamma}{2\left\|\nabla h\left(x_{0}\right)\right\|}, \frac{1}{2}\right)$. Then

$$
K \cap \frac{1}{2} B_{X} \subset T_{\beta}(x, S) \cap B_{X}+\varepsilon B_{X} .
$$

Therefore

$$
\begin{aligned}
\gamma B_{Y} & \subset \nabla h\left(x_{0}\right)\left(K \cap \frac{1}{2} B_{X}\right) \\
& \subset \nabla h\left(x_{0}\right)\left(T_{\beta}(x, S) \cap B_{X}\right)+\varepsilon \nabla h\left(x_{0}\right) B_{X} \\
& \subset \nabla h\left(x_{0}\right)\left(T_{\beta}(x, S) \cap B_{X}\right)+\frac{\gamma}{2} B_{X} .
\end{aligned}
$$


Finally let us discuss regularity for multifunctions.

DEFINITION 4.17. Let $X$ and $Y$ be Banach spaces and $F$ be a multifunction from $X$ to $Y$. We say that $F$ is regular at $\left(x_{0}, y_{0}\right) \in G r(F)$ if there exist positive constants $\varepsilon$ and $\gamma$ such that, for all $x \in x_{0}+\varepsilon B, y \in y_{0}+\varepsilon B$, and $d\left(y_{0}, F(x)\right)<\varepsilon$,

$$
d\left(x, F^{-1}(y)\right) \leq \gamma d(y, F(x)) .
$$

The following well-known lemma (see, e.g., [1,29]) will enable us to connect the regularity of a multifunction to that of a single-valued function and derive sufficient conditions for a multifunction to be regular through various of the results discussed before. We include a proof here for completeness.

LEMMA 4.18. Let $p: X \times Y \rightarrow Y$ be defined by $p(x, y)=y$. For a multifunction $F$ from $X$ to $Y$ to be regular at $\left(x_{0}, y_{0}\right) \in G r(F)$, it suffices that $p$ be regular with respect to $\operatorname{Gr}(F)$ at $\left(x_{0}, y_{0}\right)$.

Proof. Since $p$ is regular with respect to $\operatorname{Gr}(F)$ at $\left(x_{0}, y_{0}\right)$, there exist positive constants $\varepsilon$ and $\gamma$ such that, for all $(x, z) \in\left(\left(x_{0}, y_{0}\right)+\varepsilon B\right) \cap \operatorname{Gr}(F)$ and $y \in y_{0}+\varepsilon B$,

$$
d\left((x, z), \operatorname{Gr}(F) \cap p^{-1}(y)\right) \leq \gamma\|z-y\| .
$$

We may assume that $\gamma>1$. Let $\varepsilon_{1}=\varepsilon / 2$. If $\left\|x-x_{0}\right\|<\varepsilon_{1}$, $\left\|y-y_{0}\right\|<\varepsilon_{1}$, and $d\left(y_{0}, F(x)\right)<\varepsilon_{1}$, then taking the infimum with respect to $z \in F(x)$ yields

$$
\begin{aligned}
\gamma d(y, F(x)) & =\gamma \inf \{\|z-y\|: z \in F(x)\} \\
& =\gamma \inf \left\{\|z-y\|: z \in F(x),\left\|z-y_{0}\right\|<\varepsilon\right\} \\
& \geq \inf _{z \in F(x)} d\left((x, z), G r(F) \cap p^{-1}(y)\right) \\
& =\inf _{z \in F(x)} \inf \left\{\left\|x-x^{\prime}\right\|+\left\|z-y^{\prime}\right\|: x^{\prime} \in F^{-1}\left(y^{\prime}\right), y^{\prime}=y\right\} \\
& =\inf _{z \in F(x)}\left\{d\left(x, F^{-1}(y)\right)+\|z-y\|\right\} \\
& =d\left(x, F^{-1}(y)\right)+d(y, F(x)) .
\end{aligned}
$$

Thus,

$$
d\left(x, F^{-1}(y)\right) \leq(\gamma-1) d(y, F(x))
$$

Using this lemma and the various sufficient conditions that we discussed before will lead to sufficient conditions for a multifunction to be regular. For example, combining Lemma 4.18 with Theorem 4.9 we get the following theorem.

THEOREM 4.19. Let $X$ and $Y$ be Banach spaces with $\beta$-smooth norms, $F$ be a multifunction from $X$ to $Y$, and $p$ be the projection defined in Lemma 4.18. Suppose that

(i) $\mathrm{cl}\left[p\left(T_{c}\left(x_{0}, y_{0} ; G r(F)\right)\right)\right]=Y$,

(ii) there exists a nonempty compact set $K \subset Y$ and positive numbers $\alpha, \gamma$, and $\eta$ such that $\alpha<\gamma$ and

$$
\gamma B_{Y} \subset \operatorname{cl}\left\{p\left(T_{c}\left(x_{0}, y_{0} ; G r(F)\right)\right) \cap B_{X}+\alpha B_{Y}\right\}+K
$$

whenever $\left\|x-x_{0}\right\| \leq \eta$ and $\left\|y-y_{0}\right\| \leq \eta$.

Then $F$ is regular at $\left(x_{0}, y_{0}\right)$. 
Acknowledgments. We thank P. D. Loewen, A. Ioffe, L. Thibault, and the referees for their helpful comments and criticism regarding an earlier version of this paper.

\section{REFERENCES}

[1] J.-P. Aubin And H. Frankowska, Set-Valued Analysis, Birkhäuser, Boston, 1990.

[2] D. AzÉ AND C. C. CHOU, On a Newton type iterative method for solving inclusions, in Math. Oper. Res., to appear.

[3] J. M. BoRwEIN, Stability and regular points of inequality systems, J. Optim. Theory Appl., 48 (1986), pp. 9-52.

[4] —_, Epi-Lipschitz-like sets in Banach space: Theorems and examples, Nonlinear Anal., 11 (1987), pp. 1207-1217.

[5] J. M. BorweIn AND H. M. Strojwas, The hypertangent cone, Nonlinear Anal., 13 (1989), pp. 125-144.

[6] J. M. BoRWEIN AND S. FITZPATRICK, A weak Hadamard smooth renorming of $L_{1}(\Omega, \mu)$, Canad. Math. Bull., 36 (1993), pp. 407-413.

[7] - Weak-star sequential compactness and bornological limit derivatives, Convex Analysis: Special issue in celebration of R. T. Rockafellar's 60th birthday, part I, 2 (1995), pp. 59-68.

[8] J. M. BoRweIn AND A. IofFE, Proximal analysis in smooth spaces, CECM research report 93-04 (1993), to appear in Set-Valued Anal.

[9] J. M. BoRWEIN AND D. PREISS, A smooth variational principle with applications to subdifferentiability and to differentiability of convex functions, Trans. Amer. Math. Soc., 303 (1987), pp. 517-527.

[10] J. M. BORWEIN AND Q. J. ZHU, Variational analysis in non-reflexive spaces and applications to control problems with $L^{1}$ perturbations, Nonlinear Anal., 26 (1996); to appear.

[11] F. H. Clarke, Optimization and Nonsmooth Analysis, John Wiley \& Sons, New York, 1983.

[12] - Methods of Dynamic and Nonsmooth Optimization, CBMS-NSF Regional Conf. Ser. in Appl. Math., Society for Industrial and Applied Mathematics, Philadelphia, 1989.

[13] M. G. Crandall, L. C. Evans, AND P.-L. Lions, Some properties of viscosity solutions of Hamilton-Jacobi equations, Trans. Amer. Math. Soc., 282 (1984), pp. 487-502.

[14] M. G. CRANDALl, H. ISHII, AND P.-L. LIONS, User's guide to viscosity solutions of second order partial differential equations, Bull. Amer. Math. Soc. (N.S.), 27 (1992), pp. 1-67.

[15] M. G. Crandall AND P.-L. Lions, Viscosity solutions of Hamilton-Jacobi equations, Trans. Amer. Math. Soc., 277 (1983), pp. 1-42.

[16] - Hamilton-Jacobi equations in infinite dimensions, Part I. Uniqueness of viscosity solutions, J. Funct. Anal., 62 (1985), pp. 379-396; Part II. Existence of viscosity solutions, 65 (1986), pp. 368-405; Part III, 68 (1986), pp. 214-247; Part IV. Unbounded linear terms, 90 (1990), pp. 237-283; Part V, B-continuous solutions, 97 (1991), pp. 417-465.

[17] R. DeviLLE, Stability of Subdifferential of Nonconvex Functions in Banach Spaces, preprint.

[18] R. DeVILLE, G. Godefroy, AND V. ZIZLER, Smoothness and Renormings in Banach Spaces, Pitman Monographs Surveys Pure Appl. Math. 64, John Wiley Sons, New York, 1993.

[19] - A smooth variational principle with applications to Hamilton-Jacobi equations in infinite dimensions, J. Funct. Anal., 111 (1993), pp. 197-212.

[20] R. Deville And E. M. E. Haddad, The Subdifferential of the Sum of Two Functions in Banach Spaces, I. First Order Case, preprint.

[21] M. FABIAN, On class of subdifferentiability spaces of Ioffe, Nonlinear Anal., 12 (1988), pp. 63-74.

[22] W. H. Fleming AND R. W. Rishel, Deterministic and Stochastic Optimal Control, Springer-Verlag, Berlin, 1975.

[23] B. GINSBURG AND A. D. IOFFE, The maximum principle in optimal control of system governed by semilinear equations, in Proceedings of the IMA Workshop on Nonsmooth Analysis and Geometric Methods in Deterministic Optim. Control, B. S. Mordukhovich and H. T. Sussmann, eds., IMA Vol. Math. Appl., Springer-Verlag, New York, 1995.

[24] A. D. IofFe, Regular points of Lipschitz mappings, Trans. Amer. Math. Soc., 251 (1979), pp. 61-69.

[25] - On subdifferentiability spaces, Ann. New York Acad. Sci., 410 (1983), pp. 107-119.

[26] - Subdifferentiability spaces and nonsmooth analysis, Bull. Amer. Math. Soc., 10 (1984), pp. 87-90.

[27] - Necessary conditions for nonsmooth optimization, Math. Oper. Res., 9 (1984), pp. 159-189.

[28] - Calculus of Dini subdifferentials of functions and contingent derivatives of set-valued maps, Nonlinear Anal., 8 (1984), pp. 517-539.

[29] - On the local surjection property, Nonlinear Anal., 11 (1987), pp. 565-590.

[30] - Proximal analysis and approximate subdifferentials, J. London Math. Soc., 41 (1990), pp. 175-192.

[31] A. Jourani AND L. THIBAULt, Extensions of Subdifferential Calculus Rules in Banach Spaces and Applications, preprint.

[32] A. Y. KRUGER AND B. S. MoRduKhOVICH, Extremal points and Euler equations in nonsmooth optimization, Dokl. Akad. Nauk. BSSR, 24 (1980), pp. 684-687. (In Russian.) 
[33] J. Lindenstrauss And L. Tzafriri, Classical Banach Spaces II: Function Spaces, Springer-Verlag, Berlin, 1979.

[34] P. D. Loewen, Optimal Control via Nonsmooth Analysis, CRM Lecture Notes Series, Amer. Math. Soc., Summer School on Control, CRM, Université de Montréal, 1992, Amer. Math. Soc., Providence, 1993.

[35] B. MORDUKHOVICH, Complete characterization of openness, metric regularity, and Lipschitzian properties of multifunctions, Trans. Amer. Math. Soc., 340 (1993), pp. 1-35.

[36] - Maximum principle in problems of time optimal control with nonsmooth constraints, J. Appl. Math. Mech., 40 (1976), pp. 960-969.

[37] — Generalized differential calculus for nonsmooth and set-valued mappings, J. Math. Anal. Appl., 183 (1994), pp. 250-288.

[38] B. MoRduKhovich AND Y. Shao, Nonsmooth sequential analysis in Asplund spaces, Trans. Amer. Math. Soc., to appear.

[39] R. R. PHELPS, Convex Functions, Monotone Operators and Differentiability, Lecture Notes in Math. 1364, Springer-Verlag, New York, Berlin, Tokyo, 1988, 2nd ed., 1993.

[40] R. T. RockAFELLAR, Proximal subgradients, marginal values and augmented Lagrangians in nonconvex optimization, Math. Oper. Res., 6 (1981), pp. 424-436.

[41] —_, Extensions of subgradients and its applications to optimization, Nonlinear Anal., 9 (1985), pp. 665-698.

[42] L. THIBAuLT, Subdifferentials of compactly Lipschitzian vector valued functions, Ann. Math. Pura Appl. (4), 125 (1980), pp. 157-192.

[43] D. E. WARD AND J. M. BoRWEIN, Nonsmooth calculus in finite dimensions, SIAM J. Control Optim., 25 (1987), pp. 1312-1340.

[44] J. WARGA, Derivate containers, inverse functions, and controllability, in Calculus of Variations and Control Theory, D. L. Russell, ed., Academic Press, New York, 1976.

[45] —, Fat homeomorphisms and unbounded derivate containers, J. Math. Anal. Appl., 81 (1981), pp. 545-560. 